\title{
VEGETATIVE COMMUNITY DEVELOPMENT OVER 30 YEARS WITHIN MIXED PINE-HARDWOOD MINE RECLAMATION SITES IN EAST TEXAS
}

\author{
Christy L. Christian ${ }^{1}$, Brian P. Oswald, Hans M. Williams, and Kenneth W. Farrish
}

\begin{abstract}
The practice of mine reclamation aims to balance the energy needs of society with proactive environmental restoration of degraded land, and long-term studies of vegetative community development on reclaimed mine land have been invaluable in developing effective reclamation practices. This study investigated vegetative community characteristics (composition, richness, species importance) over a 30-year time frame in planted mixed pine-hardwood areas on reclaimed surface coal mine land in East Texas, United States. Reclaimed sites were compared vegetatively to unmined reference forests. A chronological pattern was shown for reclaimed community development in both understory and overstory strata. Understory community development exhibited natural patterns, while the overstory community varied with different groups of planted species. The older reclaimed sites were most similar to unmined reference sites. Dissimilarities between mined and unmined communities were also apparent; for example, the woody vine community of reference sites was much more substantial in midstory and overstory strata as compared to reclaimed sites. Overall, this study provided baseline ecological information about these plant communities that may assist land managers and researchers in furthering their development of reclamation techniques and attainment of reclamation goals.
\end{abstract}

Additional Key Words: composition, importance, lignite coal, microtopography, richness, surface mining, succession, wildlife habitat

${ }^{1}$ Christy L. Christian is a Master of Science Degree Recipient in Environmental Science, Brian P. Oswald is a Professor, Hans M. Williams is a Professor, and Kenneth W. Farrish is a Professor, Division of Environmental Science, Arthur Temple College of Forestry and Agriculture, Stephen F. Austin State University, Nacogdoches, TX 75962.

Journal American Society of Mining and Reclamation, 2016 Volume 5, Issue 2 pp 19-55

DOI: http://doi.org/10.21000/JASMR16020019 


\section{$\underline{\text { Introduction }}$}

Ecosystems are highly disturbed during surface mining for coal, a staple energy resource for the United States. Past post-mining experiences led to federal and state laws aiming to proactively mitigate environmental hazards and degradation left after mining. Reclamation of mined lands includes revegetation, which is accomplished with various land covers (commercial forest plantations, farmland, hay and pasture land, and mixed forest). Zipper et al. (2011) and Skousen and Zipper (2014) provide background information on surface coal mine reclamation, post-mining land uses and the federal Surface Mining Control and Reclamation Act of 1977 (SMCRA).

Many studies have investigated soils, vegetation, water quality, and more on unreclaimed and reclaimed mine lands both pre- and post-SMCRA (see Zipper et al., 2011 and Skousen and Zipper, 2014, for examples). Over time, reclamation strategies have changed in order to effectively address environmental issues common on mined land (e.g., acid mine drainage, erosion, lack of natural revegetation) and to achieve more successful post-mining land uses; methods intended for specific geographic application are also being developed (e.g., the Forestry Reclamation Approach (FRA) for the eastern United States) (Zipper et al., 2011; Skousen and Zipper, 2014). Long-term studies of vegetative community development on reclaimed mine land have been invaluable in developing effective reclamation practices, and several have been conducted within the United States (e.g., Brenner et al., 1984; Holl and Cairns, 1994; Holl, 2002).

In Texas, current lignite coal mine permits cover nearly 132,000 ha of land (RCT, 2015). Although no long-term studies for East Texas (Pineywoods vegetation area) were discovered during literature review, a few were conducted in east-central Texas (Post Oak Savannah/Blackland Prairie vegetation area) (Skousen et al., 1990; Gorsira and Risenhoover, 1994; Westerman, 1997). Several short-term vegetation-related studies on reclaimed mine land have been conducted in East Texas; these included research on survival and growth of various native pine species in mine soil as well as effects of fertilization rates, cover crops, ectomycorrhizal inoculation, stock type, and seed source on seedlings and young trees planted on reclaimed mine land (Bryson, 1973; Mask, 1983; Kee, 1984; Wood, 1985; Shupe, 1986; Toups, 1986; Musgraves, 1995; McGuire, 1998). However, none of these studies addressed plant communities as a whole or over time. 
The objectives of this study were to determine vegetative community characteristics (composition, richness, species importance) over a 30-year time frame (1980 to 2009) in planted mixed pine-hardwood areas on reclaimed surface coal mine land in East Texas, U.S.A. and to vegetatively compare reclaimed communities to unmined reference forests. These areas were planted with a mixture of Pinus taeda (loblolly pine) and various hardwood species. This study provided baseline ecological information about these plant communities that may assist land managers and researchers in furthering their development of reclamation techniques and attainment of reclamation goals.

\section{Methods}

\section{Study Area}

This study was conducted on Luminant Mining Company property at the Beckville mine in Panola County, Texas, United States (approximately lat $32^{\circ} 10^{\prime} \mathrm{N}$, long $94^{\circ} 20^{\prime} \mathrm{W}$ ). Post-SMCRA reclamation activities occurred within the study area over the past 30 years. The study area was characterized by irregular, gently rolling to hilly forestland; high precipitation, humidity and temperatures; acidic sand and sandy loam soils; mixed land use (includes timber, pasture, farm); and major overstory species consisting of pines, oaks, hickories, and maples (Pineywoods vegetation area) (Gould, 1962). The "subtropical" climate was characterized by annual rainfall of 42 to 46 inches (approximately 107 to $117 \mathrm{~cm}$ ), mean annual temperature of $66^{\circ} \mathrm{F}$ (approximately $19^{\circ} \mathrm{C}$ ), and mean frost free period of 230 to 245 days (Diggs et al., 2006).

Generally, pre-mine soil associations consisted of Sacul-Bowie, Fuquay-Troup, and NahatcheMantachie-Urbo, described as follows: Sacul-Bowie, "gently sloping to moderately steep, slightly acid to medium acid, loamy soils on uplands," Fuquay-Troup, "gently sloping to moderately steep, slightly acid, sandy soils on uplands," and Nahatche-Mantachie-Urbo, "nearly level, slightly acid to strongly acid, loamy to clayey soils on bottom lands" (Dolezel, 1975). A mixed overburden reclamation technique was used at this mine, whereby soil overlying coal resources was removed and set aside, coal was extracted, and the removed soil was used to re-fill excavated areas without any effort to restore pre-mining soil profile (i.e., generally, weathered surface soils became mixed with deeper, unweathered soils).

$\underline{\text { Study Sites }}$ 
Site age since establishment was the main variable used to discern trends over time in vegetative community development. Thirty-one reclaimed sites planted in mixed pine-hardwood were sampled during the 2010 and 2011 growing seasons. Study sites were randomly selected to include sites reclaimed between 1980-2009 and were grouped into six categories (i.e., 1 to 5 years, 6 to 10 years, 11 to 15 years, 16 to 20 years, 21 to 25 years, 26 to 30 years). If available, five sites were randomly chosen within each age category (at least one per year as possible). Study sites were not chosen based on site management techniques (e.g., initial cover crop, seedling type, planting density, percentage of each planted species, thinning, etc.), and, given the length of time covered by this study and frequent changes in post-SMCRA reclamation techniques, management methods may have varied among sites and were not accounted for; time since site establishment was the focus of this study. For this study, a pseudo-chronosequence was constructed using available reclaimed sites of various ages to represent a 30-year timeline. The phrase "over time" throughout this manuscript should be viewed based on the above explanation. The terms "younger," "middle-aged," and "older" were applied to sites within the following age ranges, respectively: 1 to 10 years, 11 to 20 years, and 21 to 30 years. In order to compare vegetative communities of reclaimed sites and nearby unmined forest, three reference sites were established in unmined forested land within Beckville mine property and sampled in an identical manner to reclaimed sites. For this study, it was assumed reference sites had been undisturbed for at least 30 years (amount of time for which land was controlled by mining company), and, for analysis purposes, reference sites were assigned ages of greater than 30 years. Representative photographs of study sites are included as Appendix 2.

\section{$\underline{\text { Vegetation }}$}

One plot was established in each study site using a modified-Whittaker plot design $(20$ x $50 \mathrm{~m}$ rectangular plot containing nested rectangular subplots of three sizes) (Fig. 1) (Stohlgren et al., 1995; NIISS, 2010). The 50-m side of the plot was situated parallel to the slope to ensure that vegetative samples captured as much heterogeneity as possible. Understory, midstory, and overstory strata were sampled for vegetation parameters and defined by vegetation height: understory $(\leq 1 \mathrm{~m})$, midstory $(>1 \mathrm{~m}$ to $\leq 6 \mathrm{~m})$, and overstory $(>6 \mathrm{~m})$ (Fig. 1). Vascular vegetation was identified to species, as possible, for all strata, following the USDA PLANTS 


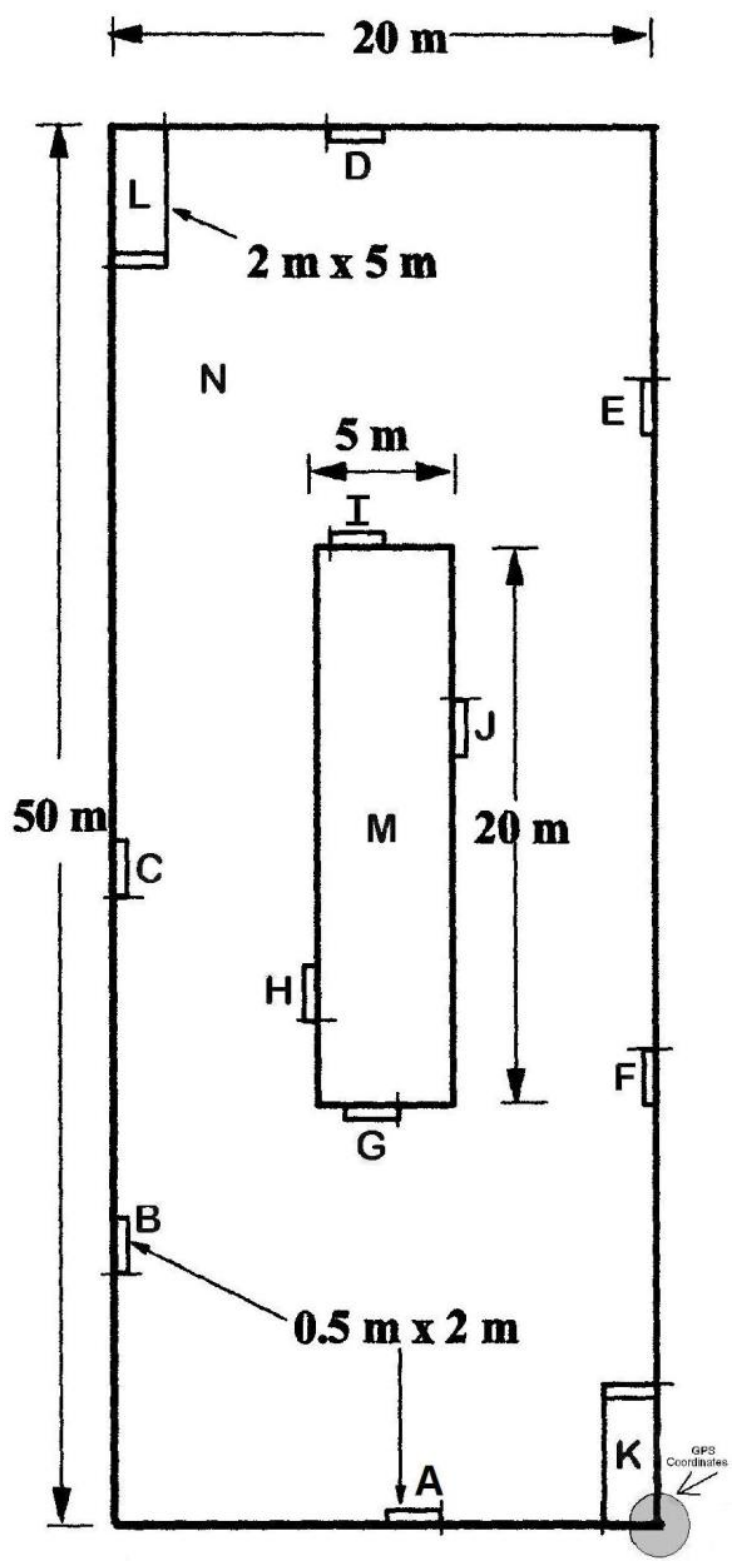

Figure 1. Layout of the modified-Whittaker plot design (Stohlgren et al., 1995). Alphabetical labels and location of GPS coordinates were added. Vegetation was sampled in subplots and strata based on vegetation height as follows: understory $(\leq 1 \mathrm{~m})$, "A" through "J," midstory ( $>1 \mathrm{~m}$ to $\leq 6 \mathrm{~m})$, "K" through "M," overstory $(>6 \mathrm{~m})$, "M" and entire plot "N".

Database for scientific name and authority (USDA, 2013). Species planted on reclamation sites varied over time (Appendix 1, Table A-1); planting years listed for each species were considered approximate and should not be viewed as absolute for data analysis. Visual estimates of species cover (\%) were recorded for understory and midstory strata, and overstory species cover was represented by basal area, which was calculated from diameter at breast height (dbh) using the 
formula $\pi^{*}(\mathrm{dbh} / 2)^{2}$. Stem counts (density) were recorded for midstory and overstory strata and converted to stems/hectare. Overstory relative density was derived from ratio of absolute species density (stems/hectare) to total stem density of site.

Species composition (i.e., presence), species richness, and species importance values (IV) were obtained from field data. Species IV are unitless numbers that indicate the overall contribution of an individual species to a community relative to all other species in the community (Barbour et al., 1999). The original definition of IV is the sum of relative cover, relative density, and relative frequency (Curtis and MacIntosh, 1951; as cited by Barbour et al., 1999), and this definition was used for midstory and overstory. For understory, IV was calculated as the sum of relative cover and relative frequency. For each species, the total species IV was calculated as the sum of understory, midstory, and overstory IV.

Data analysis included nonmetric multidimensional scaling (NMS), an ordination statistical technique performed using PC-ORD 6 statistical software (McCune and Mefford, 2011). Species codes displayed on ordination graphs are defined in Appendix 1 (Table A-2). City-block distance measures were used for two reasons: 1) the sparsity of the matrices of the datasets and 2) zeros in the dataset did not necessarily mean the numerical zero. Both Sorensen and Jaccard city-block distance measures were used to ensure that solutions obtained were similar for the two measures. In order to determine the appropriate number of axes, Autopilot was run a minimum of three times using random seeds for each of the Sorensen and Jaccard distance measures. If solutions among the Sorensen and Jaccard distance measures were similar, then Sorensen was used in the manual and final analyses. Running several different analyses ensured that a qualitatively inconsistent solution was not chosen as the final solution. Randomization tests were included to assess the strength of the data pattern. Final stress values, randomization test p-values, scree plots, and plotted ordination solutions were examined for overall qualitative consistency among all solutions. Then, a minimum of three manual NMS analyses were run using the number of axes recommended by Autopilot using Sorensen distance measures. In situations where it appeared that a different number of axes from what Autopilot recommended might be more appropriate, three manual analyses were also run using this alternative axis number. For example, in many cases, two axes were recommended, but stress values and other information indicated that three axes might also be appropriate. Mantel tests were run to compare the two axis quantities in order to determine whether or not they conveyed similar information. If the two different axis quantities provided 
similar information, the least number of axes was used in the final solution in order to simplify interpretation of results.

Further statistical analyses beyond this were not incorporated into the design of this study as its main goal was to provide basic ecological information where none existed for this type of land reclamation in this region of Texas. This study is intended as a starting point for further research. As such, statements concerning results should not be viewed in a statistical sense.

\section{$\underline{\text { Results }}$}

\section{Species Composition}

A strong chronological pattern was observed with distinct groups emerging for younger, middle-aged, and older sites along Axis 1 (axis represents sites age) (Fig. 2). Species associated with older sites were woody vines (e.g., Toxicodendron radicans (poison ivy), Parthenocissus quinquefolia (Virginia creeper)), woody shrubs (e.g., Ilex vomitoria (yaupon), and trees (e.g., Juniperus virginiana (eastern red cedar), Fraxinus pennsylvanica (green ash)) (species lines point toward older sites). Younger sites had strong association to many shade-intolerant herbaceous and grass species (e.g., Trifolium vesiculosum (arrowleaf clover), Sorghum halepense (Johnsongrass)) as well as certain oak species (e.g., Quercus lyrata (overcup oak), Quercus shumardii (Shumard's oak)) (species lines point toward younger sites). These patterns were echoed in separate analysis of understory species (Fig. 3). Ordination of overstory species indicated a chronological pattern as well (Fig. 4). Loblolly pine had strong association with older sites (species line points toward older sites), and a group of several oak species were associated with younger sites (species lines point toward younger sites) (Fig. 4). Other non-oak hardwoods were also associated with older and middle-aged sites (e.g., Celtis laevigata (sugarberry), Liquidambar styraciflua (sweetgum)) (see species lines). Anecdotally, sites reclaimed in the 1980s had an approximate planting ratio of eight or nine loblolly pine trees to each hardwood tree

(Grimes, 2010, personal communication); field data verified that planted loblolly pine stem density decreased from older sites (established in 1980s, 21 to 30 years old) to younger and middle-aged sites (established after 1980s, 1 to 20 years old (Fig. 5)).

\section{$\underline{\text { Species Richness }}$}

Generally, after 20 years, overstory richness declined while midstory richness showed some increase (Fig. 6). Understory richness was fairly variable from site to site over time (Fig. 6). 
Between reclaimed and unmined sites, mean total site richness was similar for the understory while unmined sites had higher mean richness in both midstory and overstory strata (Table 1).

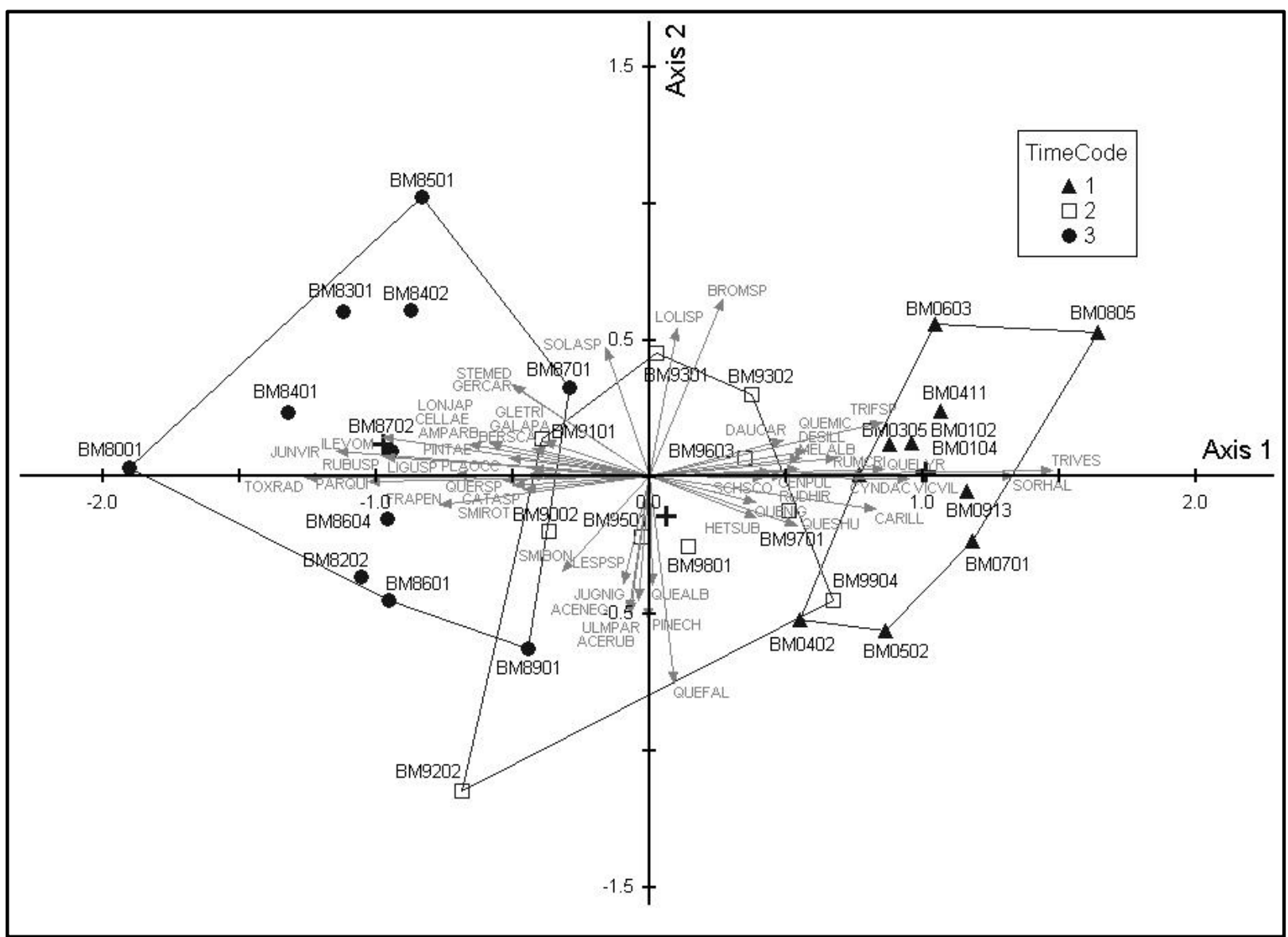

Figure 2. Nonmetric multidimensional scaling (NMS) ordination of reclaimed sites planted in mixed pine-hardwood at Beckville lignite coal surface mine (Panola County, Texas, sampled in 2010 and 2011) based on species importance values and including all vegetation strata (understory, midstory, overstory). Site legend: $1=$ sites aged 1 to 10 years; $2=$ sites aged 11 to 20 years; $3=$ sites aged 21 to 30 years. Stand name: $\mathrm{BM}=$ Beckville mixed, first two numbers indicate year of site establishment, last two numbers indicate randomly assigned site number. Sites with similar vegetation communities plotted relatively close together and dissimilar sites plotted relatively far apart. Species represented by lines and species codes (first three letters each of generic name and specific epithet); species lines represent strength (direction and magnitude) of a species' association with an axis. 


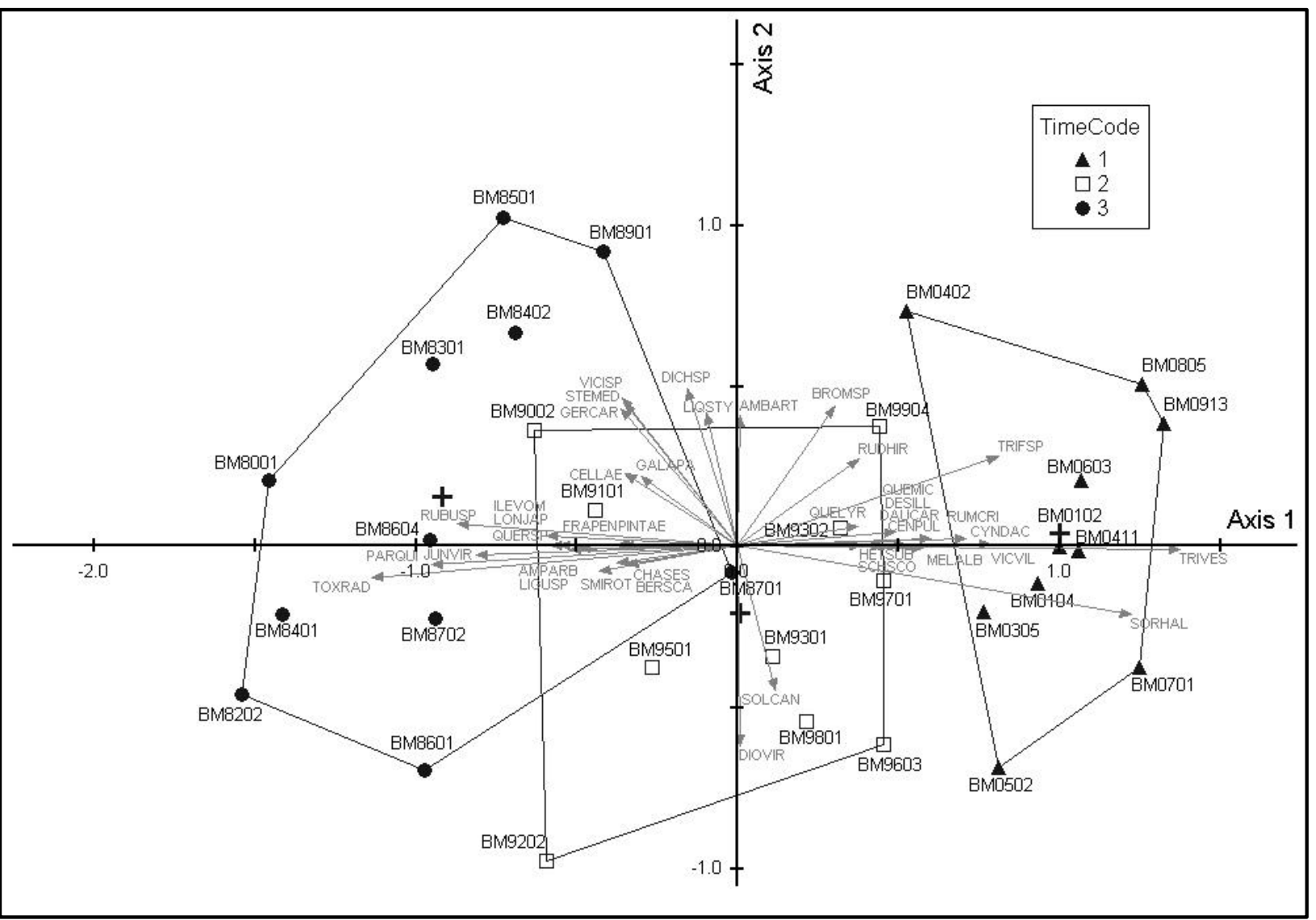

Figure 3. Nonmetric multidimensional scaling (NMS) ordination of reclaimed sites planted in mixed pine-hardwood at Beckville lignite coal surface mine (Panola County, Texas, sampled in 2010 and 2011) based on species importance values for all understory species. Site legend: $1=$ sites aged 1 to 10 years; $2=$ sites aged 11 to 20 years; $3=$ sites aged 21 to 30 years. Stand name: $\mathrm{BM}=$ Beckville mixed, first two numbers indicate year of site establishment, last two numbers indicate randomly assigned site number. Sites with similar understory vegetation communities plotted relatively close together and dissimilar sites plotted relatively far apart. Species represented by lines and species codes (first three letters each of generic name and specific epithet); species lines represent strength (direction and magnitude) of a species' association with an axis. 


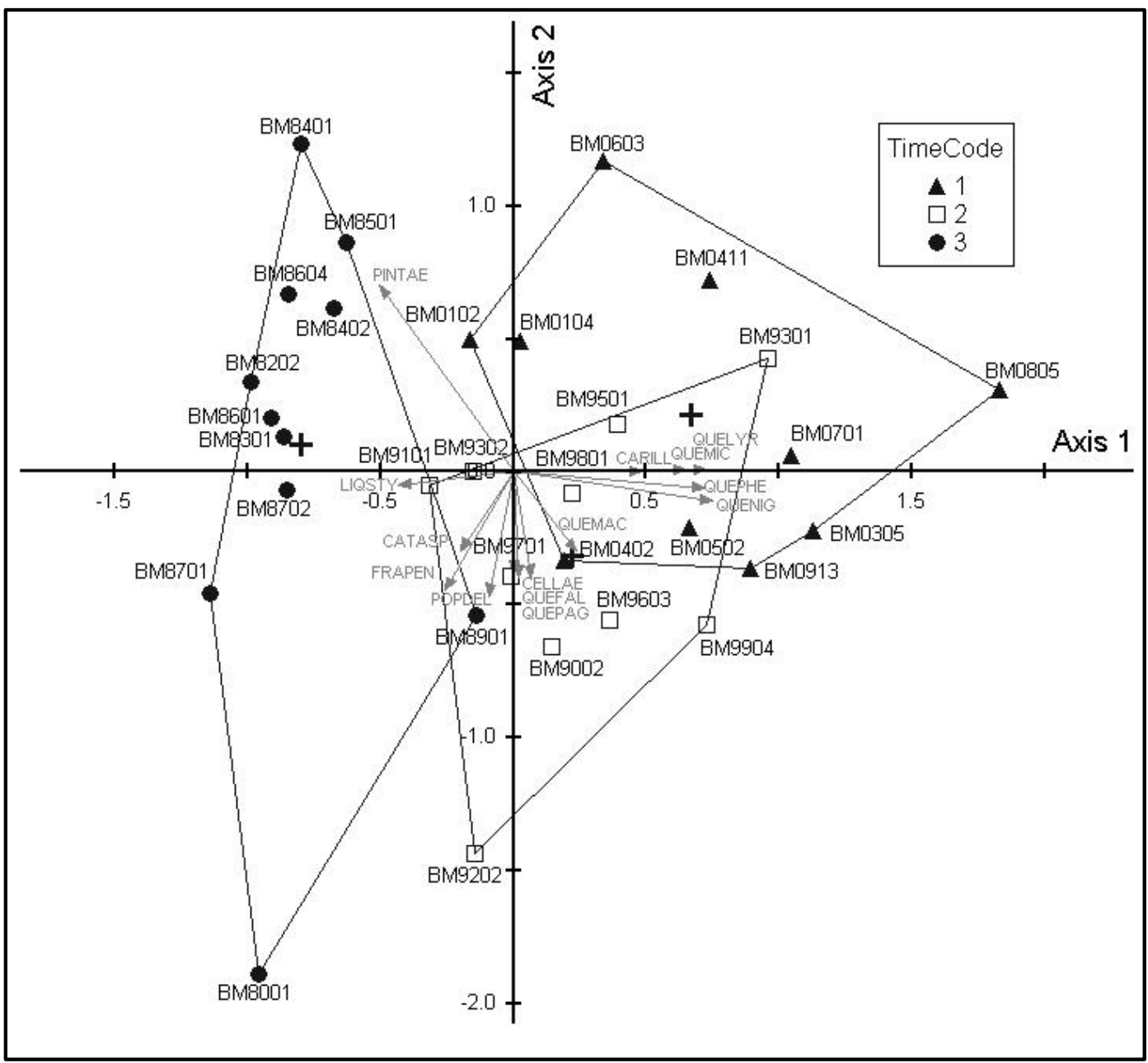

Figure 4. Nonmetric multidimensional scaling (NMS) ordination of reclaimed sites planted in mixed pine-hardwood at Beckville lignite coal surface mine (Panola County, Texas, sampled in 2010 and 2011) based on species importance values for all overstory species. Site legend: $1=$ sites aged 1 to 10 years; $2=$ sites aged 11 to 20 years; $3=$ sites aged 21 to 30 years. Stand name: $\mathrm{BM}=$ Beckville mixed, first two numbers indicate year of site establishment, last two numbers indicate randomly assigned site number. Sites with similar overstory vegetation communities plotted relatively close together and dissimilar sites plotted relatively far apart. Species represented by lines and species codes (first three letters each of generic name and specific epithet); species lines represent strength (direction and magnitude) of a species' association with an axis. 


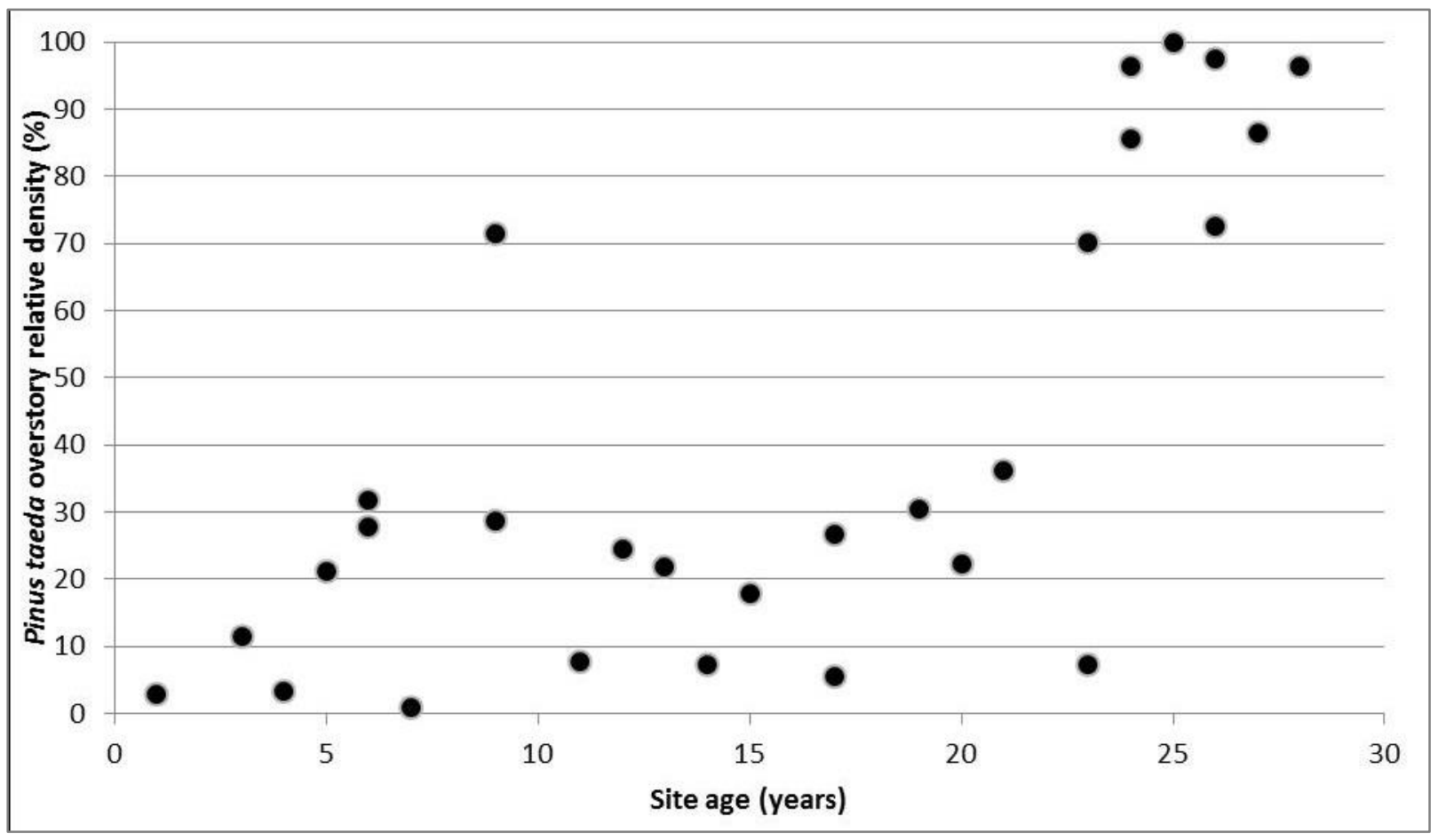

Figure 5. Overstory relative species density (\%) for Pinus taeda (loblolly pine) on reclaimed sites aged 1 to 30 years (sites established from 1980 to 2009) at Beckville lignite coal surface mine, Panola County, Texas. Reclaimed sites were planted in mixed pine-hardwood.

Species Importance Value

The ubiquitous and dominant presence of loblolly pine in multiple strata of sites of multiple ages placed it clearly as the most important species in reclaimed sites (Table 2). Other species (Baccharis halimifolia (eastern baccharis), Quercus nigra (water oak), eastern redcedar, green ash) with similar presence patterns were also important overall, albeit to a lesser degree than loblolly pine. Several oak species were of notable importance in both midstory and overstory strata along with other various trees and shrubs (sweetgum, Diospyros virginiana (common persimmon), yaupon, Morella cerifera (wax myrtle). A mixture of herbaceous, grass and vine species were most important in the understory. 
Table 1. Mean total site richness for reclaimed sites (planted in mixed pine-hardwood) and unmined forested reference sites on Beckville lignite coal surface mine in Panola County, Texas, (sampled in 2010 and 2011).

\begin{tabular}{lcccc}
\hline \multicolumn{1}{c}{ Site type } & $\begin{array}{c}\text { Understory } \\
\text { stratum }\end{array}$ & $\begin{array}{c}\text { Midstory } \\
\text { stratum }\end{array}$ & $\begin{array}{c}\text { Overstory } \\
\text { stratum }\end{array}$ & $\begin{array}{c}\text { All strata } \\
\text { combined }\end{array}$ \\
\hline $\begin{array}{l}\text { Reclaimed (planted in } \\
\text { mixed pine-hardwood) } \\
(31 \text { sites, } 1 \text { to 30 years old) }\end{array}$ & 23 & 9 & 6 & 28 \\
$\begin{array}{l}\text { Unmined forested } \\
\text { reference } \\
(3 \text { sites, }>\text { 30 years old })\end{array}$ & 20 & 21 & 14 & 32 \\
\hline
\end{tabular}

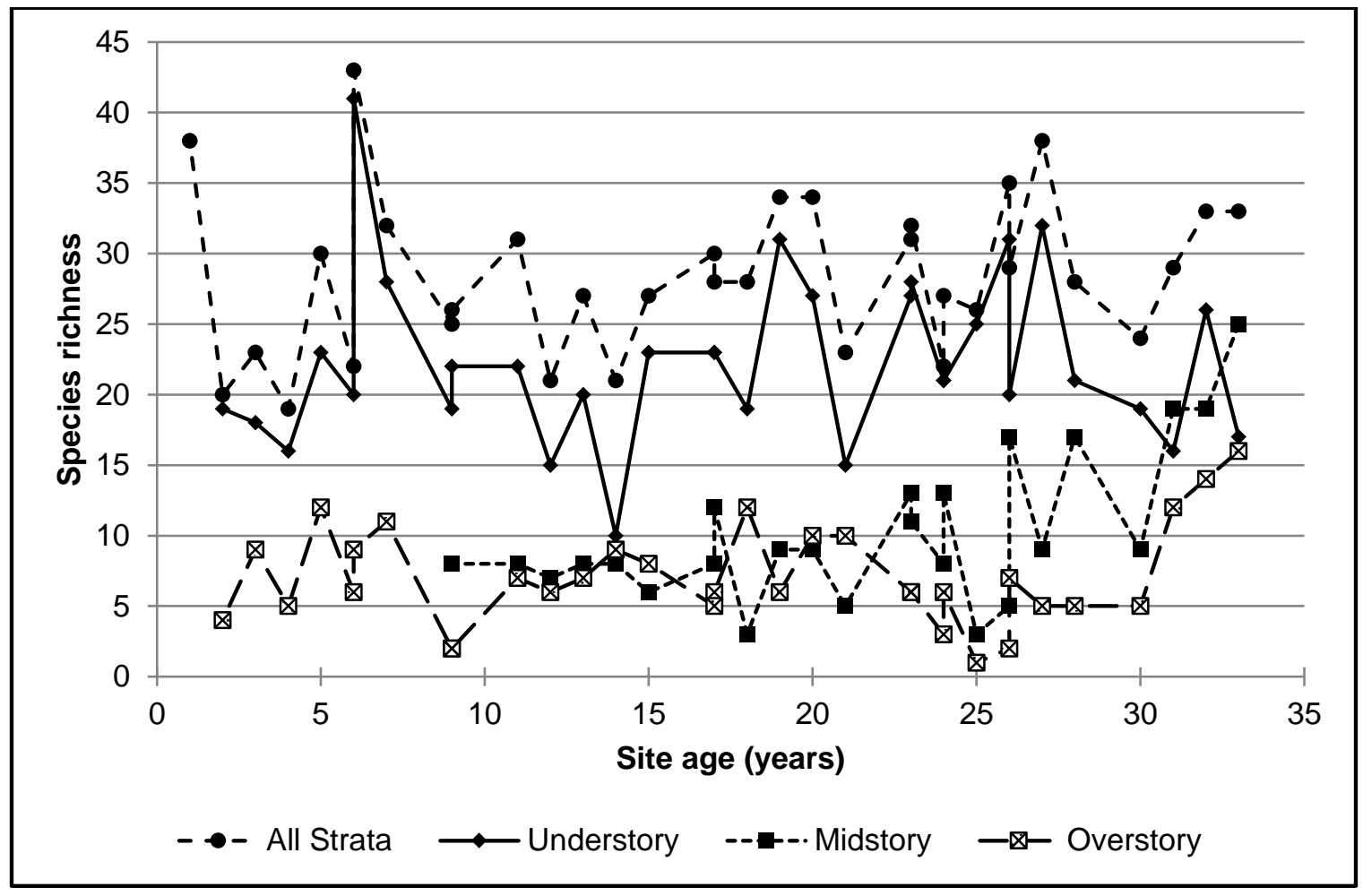

Figure 6. Species richness over time by vegetation stratum for 31 reclaimed sites aged 1 to 30 years (planted in mixed pine-hardwood) and three unmined forested reference sites at Beckville lignite coal surface mine (Panola County, Texas, sampled in 2010 and 2011). Specific age of reference sites unknown and assumed to be undisturbed for at least 30 years; reference sites graphed at age 31,32 , and 33 years. 
Table 2. Total species importance values for all sites combined for plant species observed on reclaimed sites aged 1 to 30 years (planted in mixed pine-hardwood) at Beckville lignite coal surface mine (Panola County, Texas, sampled in 2010 and 2011). Site age codes: $1=1$ to 10 years old; $2=11$ to 20 years old; $3=21$ to 30 years old. Species with total importance values of 200 or less were omitted.

\begin{tabular}{|c|c|c|c|c|c|c|}
\hline \multirow[t]{2}{*}{ Species name } & \multirow{2}{*}{$\begin{array}{c}\text { Site age } \\
\text { codes } \\
\text { where } \\
\text { observed }\end{array}$} & \multirow{2}{*}{$\begin{array}{c}\text { No. of } \\
\text { sites } \\
\text { where } \\
\text { observed }\end{array}$} & \multicolumn{3}{|c|}{$\begin{array}{l}\text { Total species importance } \\
\text { value by stratum }\end{array}$} & \multirow{2}{*}{$\begin{array}{r}\text { Total } \\
\text { species } \\
\text { importance } \\
\text { value }\end{array}$} \\
\hline & & & Understory & Midstory & Overstory & \\
\hline Pinus taeda (Loblolly pine) & $1,2,3$ & 28 & 1,029 & 1,563 & 5,591 & 8,182 \\
\hline $\begin{array}{l}\text { Baccharis halimifolia (Eastern } \\
\text { baccharis) }\end{array}$ & $1,2,3$ & 23 & 1,908 & 2,266 & 750 & 4,924 \\
\hline Quercus nigra (Water oak) & $1,2,3$ & 22 & 1,200 & 763 & 2,709 & 4,672 \\
\hline $\begin{array}{l}\text { Juniperus virginiana (Eastern } \\
\text { redcedar) }\end{array}$ & 2,3 & 19 & 1,703 & 1,804 & 616 & 4,123 \\
\hline $\begin{array}{l}\text { Fraxinus pennsylvanica (Green } \\
\text { ash) }\end{array}$ & 2,3 & 16 & 700 & 1,319 & 1,315 & 3,334 \\
\hline Vicia villosa (Winter vetch) & $1,2,3$ & 23 & 2,929 & 0 & 0 & 2,929 \\
\hline $\begin{array}{l}\text { Solidago canadensis (Canada } \\
\text { goldenrod) }\end{array}$ & $1,2,3$ & 26 & 2,922 & 0 & 0 & 2,922 \\
\hline Quercus macrocarpa (Bur oak) & $1,2,3$ & 15 & 600 & 1,129 & 1,029 & 2,758 \\
\hline Quercus phellos (Willow oak) & $1,2,3$ & 16 & 921 & 444 & 1,263 & 2,628 \\
\hline Trifolium sp. (Clover) & $1,2,3$ & 24 & 2,590 & 0 & 0 & 2,590 \\
\hline $\begin{array}{l}\text { Liquidambar styraciflua } \\
\text { (Sweetgum) }\end{array}$ & 2,3 & 13 & 700 & 761 & 1,116 & 2,576 \\
\hline $\begin{array}{l}\text { Quercus falcata (Southern red } \\
\text { oak) }\end{array}$ & $1,2,3$ & 15 & 800 & 713 & 1,049 & 2,562 \\
\hline $\begin{array}{l}\text { Quercus michauxii (Swamp } \\
\text { chestnut oak) }\end{array}$ & 1,2 & 13 & 800 & 814 & 801 & 2,415 \\
\hline $\begin{array}{l}\text { Diospyros virginiana (Common } \\
\text { persimmon) }\end{array}$ & $1,2,3$ & 13 & 1,005 & 765 & 634 & 2,405 \\
\hline Ilex vomitoria (Yaupon) & 2,3 & 14 & 1,214 & 1,079 & 102 & 2,396 \\
\hline $\begin{array}{l}\text { Quercus acutissima (Sawtooth } \\
\text { oak) }\end{array}$ & 2,3 & 10 & 515 & 436 & 1,232 & 2,184 \\
\hline $\begin{array}{l}\text { Toxicodendron radicans (Poison } \\
\text { ivy) }\end{array}$ & 2,3 & 15 & 1,609 & 405 & 100 & 2,114 \\
\hline Morella cerifera (Wax myrtle) & 2,3 & 11 & 521 & 1,447 & 105 & 2,073 \\
\hline $\begin{array}{l}\text { Rubus trivialis (Southern } \\
\text { dewberry) }\end{array}$ & $1,2,3$ & 19 & 2,003 & 0 & 0 & 2,003 \\
\hline $\begin{array}{l}\text { Sorghum halepense } \\
\text { (Johnsongrass) }\end{array}$ & 1,2 & 17 & 1,981 & 0 & 0 & 1,981 \\
\hline Quercus pagoda (Cherrybark oak) & 2,3 & 10 & 400 & 817 & 719 & 1,936 \\
\hline Quercus alba (White oak) & $1,2,3$ & 12 & 500 & 500 & 864 & 1,864 \\
\hline $\begin{array}{l}\text { Trifolium vesiculosum (Arrowleaf } \\
\text { clover) }\end{array}$ & 1,2 & 17 & 1,841 & 0 & 0 & 1,841 \\
\hline $\begin{array}{l}\text { Quercus shumardii (Shumard's } \\
\text { oak) }\end{array}$ & 1,2 & 12 & 500 & 245 & 1,039 & 1,784 \\
\hline Celtis laevigata (Sugarberry) & 2,3 & 11 & 601 & 674 & 386 & 1,661 \\
\hline Quercus lyrata (Overcup oak) & 1 & 8 & 500 & 362 & 738 & 1,600 \\
\hline Rubus sp. (Blackberry) & 2,3 & 15 & 1,549 & 0 & 0 & 1,549 \\
\hline Bromus sp.(Brome) & $1,2,3$ & 14 & 1,470 & 0 & 0 & 1,470 \\
\hline Carya illinoinensis (Pecan) & 1,2 & 11 & 200 & 416 & 644 & 1,260 \\
\hline Daucus carota (Wild carrot) & 1,2 & 12 & 1,239 & 0 & 0 & 1,239 \\
\hline
\end{tabular}


Table 2, continued.

\begin{tabular}{|c|c|c|c|c|c|c|}
\hline \multirow{2}{*}{ Species name } & \multirow{2}{*}{$\begin{array}{c}\text { Site age } \\
\text { codes } \\
\text { where } \\
\text { observed }\end{array}$} & \multirow{2}{*}{$\begin{array}{c}\text { No. of } \\
\text { sites } \\
\text { where } \\
\text { observed }\end{array}$} & \multicolumn{3}{|c|}{$\begin{array}{l}\text { Total species importance } \\
\text { value by stratum }\end{array}$} & \multirow{2}{*}{$\begin{array}{r}\text { Total } \\
\text { species } \\
\text { importance } \\
\text { value }\end{array}$} \\
\hline & & & Understory & Midstory & Overstory & \\
\hline Oxalis sp. (Woodsorrel) & $1,2,3$ & 12 & 1,223 & 0 & 0 & 1,223 \\
\hline $\begin{array}{l}\text { Prunus angustifolia (Chickasaw } \\
\text { plum) }\end{array}$ & 1 & 5 & 600 & 310 & 203 & 1,113 \\
\hline Ampelopsis arborea (Peppervine) & 2,3 & 9 & 905 & 200 & 0 & 1,105 \\
\hline Catalpa sp. (Catalpa) & 3 & 5 & 200 & 413 & 488 & 1,101 \\
\hline $\begin{array}{l}\text { Lonicera japonica (Japanese } \\
\text { honeysuckle) }\end{array}$ & 2,3 & 8 & 884 & 100 & 100 & 1,084 \\
\hline $\begin{array}{l}\text { Chaerophyllum tainturieri } \\
\text { (Hairyfruit chervil) }\end{array}$ & 2,3 & 10 & 1,036 & 0 & 0 & 1,036 \\
\hline $\begin{array}{l}\text { Desmanthus illinoensis (Illinois } \\
\text { bundleflower) }\end{array}$ & 1,2 & 10 & 1,027 & 0 & 0 & 1,027 \\
\hline Quercus sp. (Oak) & 2,3 & 10 & 1,016 & 0 & 0 & 1,016 \\
\hline $\begin{array}{l}\text { Erigeron philadelphicus } \\
\text { (Philadelphia fleabane) }\end{array}$ & 1,2 & 10 & 1,003 & 0 & 0 & 1,003 \\
\hline $\begin{array}{l}\text { Callicarpa americana (American } \\
\text { beautyberry) }\end{array}$ & 2,3 & 7 & 501 & 500 & 0 & 1,001 \\
\hline $\begin{array}{l}\text { Cynodon dactylon (Coastal } \\
\text { bermudagrass) }\end{array}$ & 1 & 9 & 990 & 0 & 0 & 990 \\
\hline Vicia sp. (Vetch) & 2,3 & 9 & 971 & 0 & 0 & 971 \\
\hline Melilotus officinalis (Sweetclover) & 1,2 & 10 & 953 & 0 & 0 & 953 \\
\hline Panicum virgatum (Switchgrass) & 1,2 & 9 & 939 & 0 & 0 & 939 \\
\hline $\begin{array}{l}\text { Schizachyrium scoparium (Little } \\
\text { bluestem) }\end{array}$ & 1 & 9 & 937 & 0 & 0 & 937 \\
\hline $\begin{array}{l}\text { Parthenocissus quinquefolia } \\
\text { (Virginia creeper) }\end{array}$ & 2,3 & 9 & 922 & 0 & 0 & 922 \\
\hline Ligustrum sp. (Privet) & 2,3 & 6 & 614 & 242 & 0 & 855 \\
\hline $\begin{array}{l}\text { Quercus muehlenbergii } \\
\text { (Chinkapin oak) }\end{array}$ & 1 & 4 & 200 & 333 & 315 & 848 \\
\hline Dichanthelium sp. (Rosette grass) & 2,3 & 8 & 835 & 0 & 0 & 835 \\
\hline Rhus copallinum (Winged sumac) & 3 & 5 & 501 & 200 & 125 & 826 \\
\hline Elymus sp. (Wildrye) & 1,3 & 8 & 813 & 0 & 0 & 813 \\
\hline $\begin{array}{l}\text { Berchemia scandens (Alabama } \\
\text { supplejack) }\end{array}$ & 2,3 & 6 & 601 & 200 & 0 & 801 \\
\hline Paspalum notatum (bahiagrass) & 1,3 & 6 & 793 & 0 & 0 & 793 \\
\hline Elaeagnus sp. (Oleaster) & 2,3 & 3 & 303 & 432 & 0 & 735 \\
\hline $\begin{array}{l}\text { Platanus occidentalis (American } \\
\text { sycamore) }\end{array}$ & 3 & 5 & 100 & 300 & 314 & 714 \\
\hline $\begin{array}{l}\text { Ambrosia artemisiifolia (Annual } \\
\text { ragweed) }\end{array}$ & 2,3 & 9 & 703 & 0 & 0 & 703 \\
\hline $\begin{array}{l}\text { Eupatorium serotinum } \\
\text { (Lateflowering thoroughwort) }\end{array}$ & 2,3 & 7 & 702 & 0 & 0 & 702 \\
\hline Ulmus parvifolia (Chinese elm) & 2,3 & 4 & 141 & 300 & 223 & 664 \\
\hline $\begin{array}{l}\text { Lespedeza cuneata (Sericea } \\
\text { lespedeza) }\end{array}$ & 3 & 6 & 664 & 0 & 0 & 664 \\
\hline $\begin{array}{l}\text { Geranium carolinianum (Carolina } \\
\text { geranium) }\end{array}$ & 2,3 & 6 & 625 & 0 & 0 & 625 \\
\hline Trifolium repens (White clover) & 2,3 & 6 & 624 & 0 & 0 & 624 \\
\hline $\begin{array}{l}\text { Gleditsia triacanthos } \\
\text { (Honeylocust) }\end{array}$ & 3 & 4 & 400 & 200 & 0 & 600 \\
\hline
\end{tabular}


Table 2, continued.

\begin{tabular}{|c|c|c|c|c|c|c|}
\hline \multirow{2}{*}{ Species name } & \multirow{2}{*}{$\begin{array}{c}\text { Site age } \\
\text { codes } \\
\text { where } \\
\text { observed }\end{array}$} & \multirow{2}{*}{$\begin{array}{c}\text { No. of } \\
\text { sites } \\
\text { where } \\
\text { observed }\end{array}$} & \multicolumn{3}{|c|}{$\begin{array}{c}\text { Total species importance } \\
\text { value by stratum }\end{array}$} & \multirow{2}{*}{$\begin{array}{c}\text { Total } \\
\text { species } \\
\text { importance } \\
\text { value } \\
\end{array}$} \\
\hline & & & Understory & Midstory & Overstory & \\
\hline Quercus texana (Nuttall's oak) & 2 & 2 & 0 & 200 & 360 & 560 \\
\hline $\begin{array}{l}\text { Stellaria media (Common } \\
\text { chickweed) }\end{array}$ & 3 & 5 & 554 & 0 & 0 & 554 \\
\hline $\begin{array}{l}\text { Trifolium incarnatum (Crimson } \\
\text { clover) }\end{array}$ & $1,2,3$ & 5 & 503 & 0 & 0 & 503 \\
\hline $\begin{array}{l}\text { Heterotheca subaxillaris } \\
\text { (Camphorweed) }\end{array}$ & 1 & 5 & 503 & 0 & 0 & 503 \\
\hline Acer rubrum (Red maple) & 2,3 & 2 & 100 & 300 & 102 & 502 \\
\hline $\begin{array}{l}\text { Verbena brasiliensis (Brazilian } \\
\text { vervain) }\end{array}$ & 1,2 & 5 & 501 & 0 & 0 & 501 \\
\hline $\begin{array}{l}\text { Centaurium pulchellum (Branched } \\
\text { centaury) }\end{array}$ & 1 & 5 & 501 & 0 & 0 & 501 \\
\hline $\begin{array}{l}\text { Rudbeckia hirta (Blackeyed } \\
\text { Susan) }\end{array}$ & 1 & 5 & 500 & 0 & 0 & 500 \\
\hline $\begin{array}{l}\text { Smilax rotundifolia (Common } \\
\text { greenbrier) }\end{array}$ & 3 & 3 & 300 & 200 & 0 & 500 \\
\hline Ulmus sp. (Elm) & 2,3 & 4 & 427 & 0 & 0 & 427 \\
\hline $\begin{array}{l}\text { Galium aparine (Catchweed } \\
\text { bedstraw) }\end{array}$ & 3 & 4 & 408 & 0 & 0 & 408 \\
\hline Ilex opaca (American holly) & 2,3 & 4 & 203 & 200 & 0 & 403 \\
\hline Lespedeza sp. (Lespedeza) & $1,2,3$ & 4 & 401 & 0 & 0 & 401 \\
\hline Salix nigra (Black willow) & 3 & 3 & 0 & 141 & 205 & 347 \\
\hline $\begin{array}{l}\text { Lolium perenne (Perennial } \\
\text { ryegrass) }\end{array}$ & 1,3 & 3 & 334 & 0 & 0 & 334 \\
\hline Lolium sp. (Ryegrass) & 1,3 & 3 & 303 & 0 & 0 & 303 \\
\hline $\begin{array}{l}\text { Andropogon glomeratus (Bushy } \\
\text { bluestem) }\end{array}$ & 1,2 & 3 & 300 & 0 & 0 & 300 \\
\hline $\begin{array}{l}\text { Asclepias viridis (Green } \\
\text { milkweed) }\end{array}$ & 2,3 & 3 & 300 & 0 & 0 & 300 \\
\hline Cirsium sp. (Thistle) & 1,2 & 3 & 300 & 0 & 0 & 300 \\
\hline Pinus echinata (Shortleaf pine) & 1,2 & 2 & 0 & 0 & 246 & 246 \\
\hline Vitis rotundifolia (Muscadine) & 3 & 2 & 208 & 0 & 0 & 208 \\
\hline Quercus virginiana (Live oak) & 2 & 2 & 0 & 100 & 105 & 205 \\
\hline $\begin{array}{l}\text { Ipomoea cordatotriloba (Cotton } \\
\text { morningglory) }\end{array}$ & 2 & 2 & 204 & 0 & 0 & 204 \\
\hline $\begin{array}{l}\text { Zanthoxylum clava-herculis } \\
\text { (Hercules' club) }\end{array}$ & 3 & 1 & 0 & 100 & 104 & 204 \\
\hline $\begin{array}{l}\text { Helianthus maximiliani } \\
\text { (Maximilian sunflower) }\end{array}$ & 1 & 1 & 203 & 0 & 0 & 203 \\
\hline Acer negundo (Boxelder) & 2 & 1 & 100 & 0 & 103 & 203 \\
\hline Solanum sp. (Nightshade) & 3 & 2 & 202 & 0 & 0 & 202 \\
\hline Paspalum sp. (Crowngrass) & 1 & 2 & 201 & 0 & 0 & 201 \\
\hline Erigeron sp. (Fleabane) & 3 & 2 & 201 & 0 & 0 & 201 \\
\hline
\end{tabular}




\section{$\underline{\text { Reference Sites }}$}

With regard to reclaimed sites, the unmined forested reference sites were most similar to older reclaimed sites, but, even so, dissimilarities between mined and unmined forest communities were quite apparent (Fig. 7 - 9). Several of the same woody vine species were important in the understory of reclaimed and reference sites, but vines in the midstory and overstory were rare in reclaimed sites and common in reference sites. Based on species importance in the overstory stratum, species differentiating reference from mined included tree species sugarberry, Ulmus alata (winged elm), Ulmus americana (American elm), and Carya cordiformis (bitternut hickory) and vine species Lonicera japonica (Japanese honeysuckle), Berchemia scandens (Alabama supplejack), Virginia creeper, and poison ivy (Tables 2 \& 3). Several older reclaimed sites contained understory herbaceous species not observed in reference sites. Green ash was similarly important in older reclaimed and reference sites in the understory and midstory strata but not present in the overstory of reference sites; sweetgum was important in all strata of both reclaimed and reference communities. Winged elm was observed occasionally in reclaimed sites but was consistently important in midstory and overstory of reference sites.

At the site level, reference sites exhibited greater midstory and overstory richness than older reclaimed sites but similar understory and overall richness (Table 2, Fig. 7). Out of a combined richness of 155 species for reclaimed and reference sites, 15 species were exclusively observed in reference sites and 39 species were found in both reclaimed and reference sites (Table 3). Based on importance values, reference sites were forest communities with well-developed physical structure illustrated by several tree, vine, and shrub species contributing to all three strata (Table 3). Dominant species included the trees sweetgum, winged elm, water oak, and sugarberry and six different vines. 


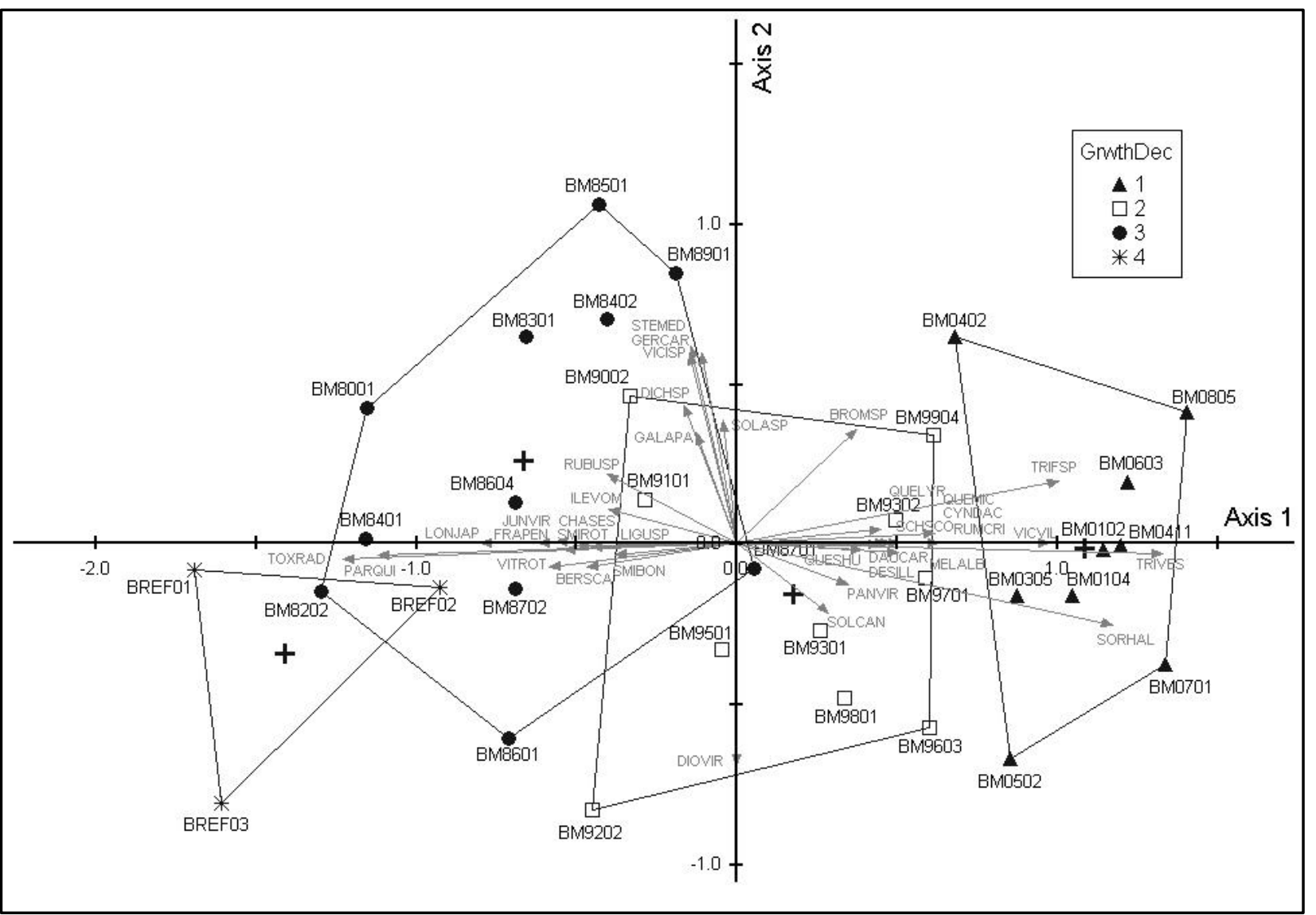

Figure 7. Nonmetric multidimensional scaling (NMS) ordination of reclaimed sites planted in mixed pine-hardwood and unmined forested reference sites at Beckville lignite coal surface mine (Panola County, Texas, sampled in 2010 and 2011) based on species importance values for all understory species. Site legend: $1=$ sites aged 1 to 10 years; $2=$ sites aged 11 to 20 years; $3=$ sites aged 21 to 30 years; $4=$ reference sites. Stand name: $\mathrm{BM}=$ Beckville mixed, $\mathrm{BREF}=\mathrm{Beckville}$ reference, first two numbers indicate year of site establishment; last two numbers indicate randomly assigned site number. Sites with similar understory vegetation communities plotted relatively close together and dissimilar sites plotted relatively far apart. Species represented by lines and species codes (first three letters each of generic name and specific epithet); species lines represent strength (direction and magnitude) of a species' association with an axis. 


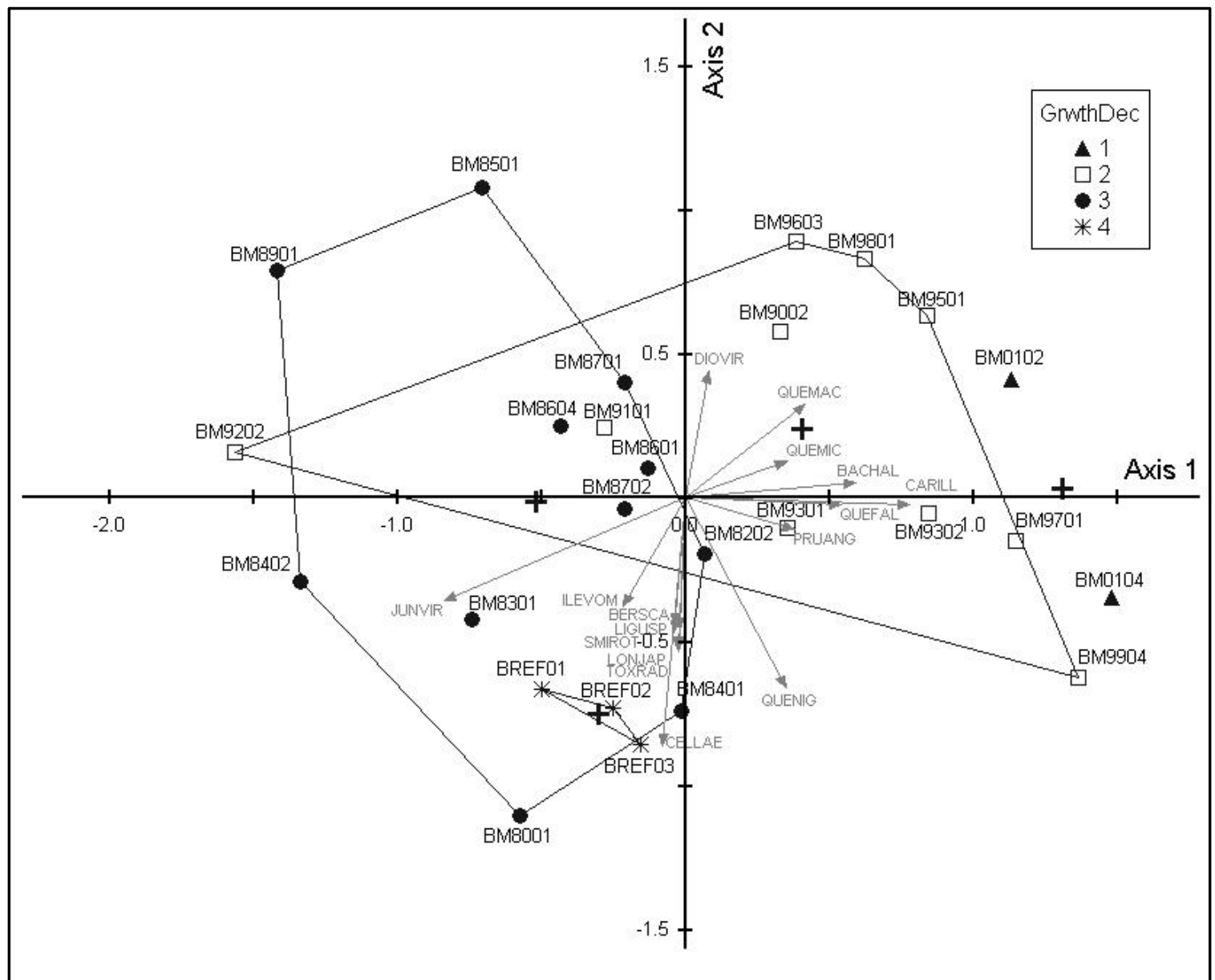

Figure 8. Nonmetric multidimensional scaling (NMS) ordination of reclaimed sites planted in mixed pine-hardwood and unmined forested reference sites at Beckville lignite coal surface mine (Panola County, Texas, sampled in 2010 and 2011) based on species importance values for all midstory species. Site legend: $1=$ sites aged 1 to 10 years; 2 $=$ sites aged 11 to 20 years; $3=$ sites aged 21 to 30 years; $4=$ reference sites. Stand name: $\mathrm{BM}=$ Beckville mixed, $\mathrm{BREF}=$ Beckville reference, first two numbers indicate year of site establishment; last two numbers indicate randomly assigned site number. Sites with similar midstory vegetation communities plotted relatively close together and dissimilar sites plotted relatively far apart. Species represented by lines and species codes (first three letters each of generic name and specific epithet); species lines represent strength (direction and magnitude) of a species' association with an axis. 


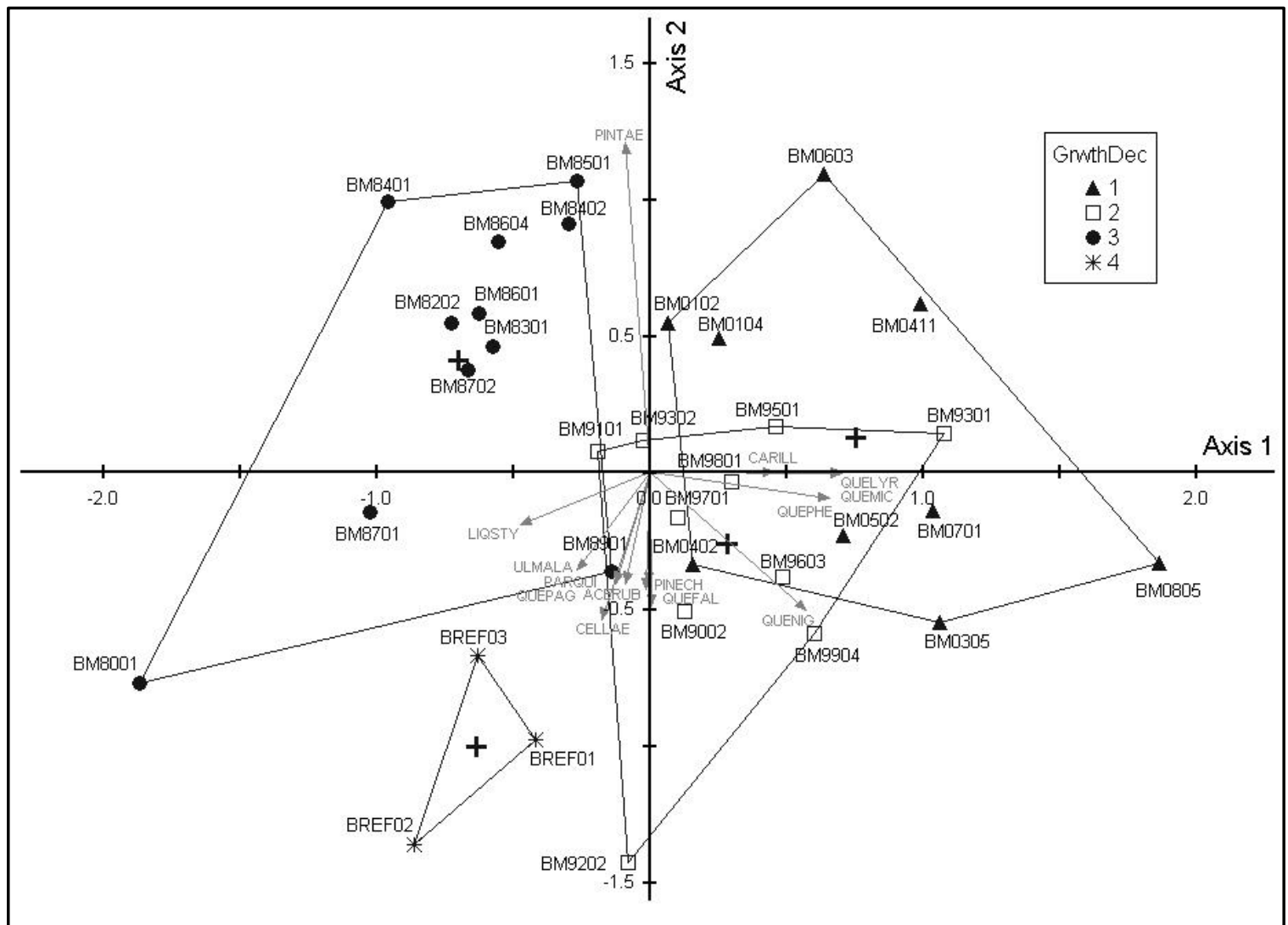

Figure 9. Nonmetric multidimensional scaling (NMS) ordination of reclaimed sites planted in mixed pine-hardwood and unmined forested reference sites at Beckville lignite coal surface mine (Panola County, Texas, sampled in 2010 and 2011) based on species importance values for all overstory species. Site legend: $1=$ sites aged 1 to 10 years; $2=$ sites aged 11 to 20 years; $3=$ sites aged 21 to 30 years; $4=$ reference sites. Stand name: $\mathrm{BM}=$ Beckville mixed, $\mathrm{BREF}=$ Beckville reference, first two numbers indicate year of site establishment; last two numbers indicate randomly assigned site number. Sites with similar overstory vegetation communities plotted relatively close together and dissimilar sites plotted relatively far apart. Species represented by lines and species codes (first three letters each of generic name and specific epithet); species lines represent strength (direction and magnitude) of a species' association with an axis. 
Table 3. Total species importance values for all sites combined for plant species observed on unmined forested reference sites (undisturbed for at least 30 years) at Beckville lignite coal surface mine (Panola County, Texas, sampled in 2010 and 2011). Species observed in both reclamation sites (planted in mixed pine-hardwood, age range 1 to 30 years) and reference sites are indicated.

\begin{tabular}{|c|c|c|c|c|c|}
\hline \multirow{2}{*}{ Species name } & \multirow{2}{*}{$\begin{array}{l}\text { Observed in } \\
\text { both } \\
\text { reclaimed \& } \\
\text { reference } \\
\text { sites }\end{array}$} & \multicolumn{3}{|c|}{$\begin{array}{c}\text { Total species importance value } \\
\text { by stratum }\end{array}$} & \multirow{2}{*}{$\begin{array}{c}\text { Total } \\
\text { species } \\
\text { importance } \\
\text { value }\end{array}$} \\
\hline & & Understory & Midstory & Overstory & \\
\hline Liquidambar styraciflua (Sweetgum) & $\cdot$ & 300 & 308 & 448 & 1,056 \\
\hline Lonicera japonica (Japanese honeysuckle) & - & 343 & 382 & 200 & 925 \\
\hline Ulmus alata (Winged elm) & - & 101 & 453 & 327 & 881 \\
\hline Toxicodendron radicans (Poison ivy) & - & 424 & 211 & 200 & 835 \\
\hline Quercus nigra (Water oak) & • & 100 & 300 & 360 & 760 \\
\hline $\begin{array}{l}\text { Parthenocissus quinquefolia (Virginia } \\
\text { creeper) }\end{array}$ & - & 332 & 200 & 200 & 732 \\
\hline Celtis laevigata (Sugarberry) & - & 102 & 316 & 305 & 724 \\
\hline Juniperus virginiana (Eastern redcedar) & - & 201 & 314 & 207 & 722 \\
\hline Fraxinus pennsylvanica (Green ash) & - & 317 & 354 & 0 & 671 \\
\hline Berchemia scandens (Alabama supplejack) & - & 222 & 200 & 200 & 622 \\
\hline Vitis rotundifolia (Muscadine) & - & 300 & 200 & 100 & 600 \\
\hline Ilex vomitoria (Yaupon) & • & 114 & 475 & 0 & 588 \\
\hline Quercus pagoda (Cherrybark oak) & - & 0 & 211 & 238 & 449 \\
\hline Smilax bona-nox (Saw greenbrier) & - & 310 & 112 & 0 & 422 \\
\hline Ulmus americana (American elm) & - & 0 & 211 & 207 & 417 \\
\hline Carya cordiformis (Bitternut hickory) & & 0 & 200 & 209 & 409 \\
\hline Quercus alba (White oak) & - & 100 & 200 & 102 & 402 \\
\hline Pinus taeda (Loblolly pine) & - & 0 & 0 & 344 & 344 \\
\hline Ligustrum sp. (Privet) & - & 200 & 132 & 0 & 332 \\
\hline Quercus falcata (Southern red oak) & • & 0 & 100 & 222 & 322 \\
\hline $\begin{array}{l}\text { Callicarpa americana (American } \\
\text { beautyberry) }\end{array}$ & - & 100 & 214 & 0 & 314 \\
\hline Sassafras albidum (Sassafras) & & 100 & 100 & 108 & 308 \\
\hline Chionanthus virginicus (White fringetree) & & 100 & 100 & 106 & 306 \\
\hline Ampelopsis arborea (Peppervine) & • & 105 & 100 & 100 & 305 \\
\hline Smilax rotundifolia (Common greenbrier) & • & 100 & 203 & 0 & 303 \\
\hline $\begin{array}{l}\text { Diospyros virginiana (Common } \\
\text { persimmon) }\end{array}$ & • & 200 & 0 & 102 & 302 \\
\hline $\begin{array}{l}\text { Chasmanthium sessiliflorum (Longleaf } \\
\text { woodoats) }\end{array}$ & - & 214 & 0 & 0 & 214 \\
\hline Acer rubrum (Red maple) & • & 0 & 100 & 105 & 205 \\
\hline Prunus serotina (Black cherry) & & 0 & 100 & 105 & 205 \\
\hline Acer barbatum (Florida maple) & & 101 & 103 & 0 & 204 \\
\hline Dichanthelium sp. (Rosette grass) & • & 201 & 0 & 0 & 201 \\
\hline Morella cerifera (Wax myrtle) & - & 100 & 100 & 0 & 200 \\
\hline Morus rubra (Red mulberry) & & 100 & 100 & 0 & 200 \\
\hline Nandina domestica (Sacred bamboo) & & 100 & 100 & 0 & 200 \\
\hline Sideroxylon lanuginosum (Chittamwood) & & 100 & 100 & 0 & 200 \\
\hline Fraxinus americana (White ash) & & 0 & 0 & 198 & 198 \\
\hline Rubus trivialis (Southern dewberry) & $\bullet$ & 109 & 0 & 0 & 109 \\
\hline Pinus echinata (Shortleaf pine) & - & 0 & 0 & 108 & 108 \\
\hline Solidago canadensis (Canada goldenrod) & - & 101 & 0 & 0 & 101 \\
\hline
\end{tabular}


Table 3, continued.

\begin{tabular}{|c|c|c|c|c|c|}
\hline \multirow{2}{*}{ Species name } & \multirow{2}{*}{$\begin{array}{l}\text { Observed in } \\
\text { both } \\
\text { reclaimed \& } \\
\text { reference } \\
\text { sites }\end{array}$} & \multicolumn{3}{|c|}{$\begin{array}{c}\text { Total species importance value } \\
\text { by stratum }\end{array}$} & \multirow{2}{*}{$\begin{array}{c}\text { Total } \\
\text { species } \\
\text { importance } \\
\text { value }\end{array}$} \\
\hline & & Understory & Midstory & Overstory & \\
\hline Oxalis sp. (Woodsorrel) & $\cdot$ & 101 & 0 & 0 & 101 \\
\hline Rubus sp. & - & 100 & 0 & 0 & 100 \\
\hline Ambrosia artemisiifolia & - & 100 & 0 & 0 & 100 \\
\hline Cornus florida & & 0 & 100 & 0 & 100 \\
\hline Hypericum hypericoides & - & 100 & 0 & 0 & 100 \\
\hline Ilex ораса & - & 0 & 100 & 0 & 100 \\
\hline Lespedeza cuneata & - & 100 & 0 & 0 & 100 \\
\hline Paspalum sp. & - & 100 & 0 & 0 & 100 \\
\hline Polystichum acrostichoides & & 100 & 0 & 0 & 100 \\
\hline Prunus caroliniana & - & 100 & 0 & 0 & 100 \\
\hline Quercus stellata & • & 0 & 100 & 0 & 100 \\
\hline Smilax glauca & & 0 & 100 & 0 & 100 \\
\hline Smilax laurifolia & & 100 & 0 & 0 & 100 \\
\hline Viburnum rufidulum & & 0 & 100 & 0 & 100 \\
\hline Vitis aestivalis & & 0 & 100 & 0 & 100 \\
\hline
\end{tabular}

\section{Discussion}

A chronological pattern was shown for reclaimed community development overall, specifically echoed in both understory and overstory. However, the trends over time in understory and overstory were due to different reasons. Aside from planted tree species, understory species were observed to shift from shade-intolerant herbaceous and grass species in younger sites to more diverse groups of shade-tolerant herbaceous species, shrubs, and woody vines in older sites; this chronological trend was illustrative of a natural development of the community. Natural succession, which produces diversity and stability over time in disturbed ecosystems, is important in mine reclamation because volunteer colonization of native species will produce plant communities that will have long-term stability in a given locale's climate and be most beneficial to native wildlife in terms of food and cover (Brenner et al., 1984).

When considering the overstory, the main influence on chronological patterns was shown to be human design, i.e., a variety of oaks was planted in younger sites while an abundance of loblolly pine along with some nonoak hardwoods was planted in older sites. For this reason, comparison of reclaimed sites along a temporal continuum was confounded. Essentially, three age-associated overstory communities were revealed (Fig. 2). Older sites largely resembled loblolly pine plantations with a few other large hardwood tree individuals present. Middle-aged sites exhibited 
a diverse mixture of loblolly pine, oaks and a few other hardwoods with dominance shared by loblolly pine and several oaks. Younger sites were generally observed to contain several oaks and loblolly pine with a few other tree species. Several younger sites were even dominated by loblolly pine and water oak only. The shade-intolerant shrub eastern baccharis was also a substantial component of some younger sites. The overstory community of younger sites was altered from middle-aged by 1) absence or near-absence of Quercus acutissima (sawtooth oak), green ash, Catalpa sp. (catalpa), eastern red cedar, and sweetgum, 2) lessened abundance of Quercus falcata (southern red oak) and Quercus pagoda (cherrybark oak), and 3) the substantial addition of overcup oak and Carya illinoinensis (pecan). For these reasons, when reclaimed younger sites reach age 30 , their overstory communities will likely not resemble the older or middle-aged sites of this study; however, in the understory and midstory, it is reasonable to expect that a succession from shade-intolerant herbaceous and grass species to a diverse mixture of shade-tolerant herbaceous species, shrubs and woody vines will occur, as demonstrated by understory composition patterns of younger and older reclaimed sites.

The notable presence of woody vines in reclaimed plant communities is encouraging. Of 39 species occurring in both reclaimed and reference sites, nine are woody vines; while found in all strata of reference sites, vines were generally only observed in the understory of reclaimed sites, which was likely due to lack of time for fuller development of the vine component. In the future, reclaimed communities will likely see movement of woody vines into the midstory and overstory. The presence of volunteer vine species is indicative of development of vertical structure in the community. An earlier study conducted on reclaimed woodland sites aged 3 to 11 years in eastcentral Texas (Gould's (1962) Post Oak Savannah) noted lack of vertical structure ("layering”) in those sites and uncertainty about time requirements for vertical structure resembling that in native woodlands to form (Gorsira and Risenhoover, 1994). This study demonstrated that a middle (midstory) vegetation layer developed around age 10 and that vine species commonly contributing to vertical forest structure in East Texas were colonizing reclaimed sites during the second decade after establishment. Gorsira and Risenhoover (1994) also noted the importance of evergreen species as cover for wildlife species in winter. In this study, evergreen species such as yaupon, eastern red cedar, and Smilax spp. were observed to be both present and fairly important in understory and midstory strata of reclaimed sites. 
Given that the forest communities of reference sites had developed without apparent disturbance for at least three decades, it was reasonable to observe the differences noted between reclaimed and reference. An earlier study by Holl (2002) concluded that, although the oldest reclaimed sites (35 years old) had some resemblance to unmined reference sites, the oldest and reference sites still differed substantially. Reference communities possessed species composition and physical structure representative of the East Texas forest ecosystem. The effects of human design were apparent in observations of greater midstory and overstory richness in reference sites than in older reclaimed sites, where loblolly pine was dominant due to planting procedures. However, four of the same tree species (water oak, green ash, sweetgum, eastern red cedar) were observed to have high importance in both reclaimed and reference sites. In time, loblolly pine dominance may lessen as the pines age and allow other large tree species to be greater contributors to the older reclaimed site communities.

None of the 15 species (10 trees, one shrub, three vines, and one fern) observed exclusively in reference sites were consistent residents across sites or strata, indicating that these are less common forest species (Table 3). However, their presence indicated that they fill certain environmental niches and contribute to greater community diversity. Several of the trees are smaller-stature species that would be expected to do well in the midstory and lower overstory strata (e.g., Cornus florida (flowering dogwood), Morus rubra (red mulberry)), and the vines were of some of the same genera found in reclaimed sites. Holl (2002) likewise observed that less common forest species were not colonizing reclaimed mine sites three decades after establishment although present in nearby unmined forested sites. The future presence of these less common forest species in reclaimed sites would indicate improving diversity.

Past concern with reclamation activities involved soil compaction and subsequent "arrested succession" that prevents return of mined land to forest. Creation of microtopography using the end-dump method within the reclaimed landscape is suggested as a remedy for arrested succession in reclaimed areas and to encourage increased vegetative diversity (Gilland and McCarthy, 2014). Soil compaction was empirically demonstrated to not be an issue on these sites, and "arrested succession" as described by Gilland and McCarthy (2014) was not observed; bulk density values ranged from 1.00 to $1.35 \mathrm{~g} / \mathrm{cm}^{3}$ for reclaimed and 1.04 to $1.10 \mathrm{~g} / \mathrm{cm}^{3}$ for reference, which are favorable values for root growth (Christian, 2013). However, incorporation of greater microtopography during establishment of reclamation sites may increase plant species diversity 
on younger reclaimed sites and form diverse forest plant communities in an even more expedient manner. Provision of a greater variety of topographic positions may, for example, encourage eventual colonization of some of the tree species observed exclusively in reference sites (e.g., bitternut hickory, Sassafras albidum (sassafras)). This method may also help control erosion in newly planted sites by encouraging plants to occupy more physical space using the greater abundance of unique topographic positions.

Overall, reclamation was effective in achieving diverse plant communities. Resampling of younger sites after another 10 and 20 years of growth will give insight to successional

development. Incorporation of microtopography during reclamation site establishment may increase diversity of the early reclaimed community and expedite the formation of a diverse forest community. Direct assessment of how plant communities developed over time was not possible for this study due to variation in reclamation techniques over the past 40 years (e.g., tree species selection). As newer methods such as the Forestry Reclamation Approach (Skousen and Zipper, 2014) are more widely implemented and other reclamation techniques that have been successful thus far continue to be used, the sample size of sites that share identical reclamation methods will increase. Temporal comparisons can then be made for more accurate ascertainment of vegetation community development over time.

\section{Acknowledgements}

Funding was provided by the Luminant Environmental Research Program. The authors gratefully acknowledge Luminant Generation Company LLC and the Division of Environmental Science, Arthur Temple College of Forestry and Agriculture, Stephen F. Austin State University for their support of this research and Melissa Bozarth and Brittany Compton for their assistance in the field. This manuscript was greatly improved by the invaluable comments of several anonymous reviewers.

\section{$\underline{\text { Literature Cited }}$}

Barbour, M.G., J.H. Burk, W.D. Pitts, F.S. Gilliam, and M.W. Schwartz. 1999. Terrestrial Plant Ecology. $3^{\text {rd }}$ ed. Benjamin/Cummings, Menlo Park. 649 p. 
Brenner, F.J., M. Werner and J. Pike. 1984. Ecosystem development and natural succession in surface coal mine reclamation. Minerals and the Environment 6: 10-22. http://dx.doi.org/10.1007/BF02072661

Bryson, H.L., Jr. 1973. Early survival and total height, and foliar analyses of eleven tree species grown on strip-mine spoils in Freestone County, Texas. MS thesis. Stephen F. Austin State University, Nacogdoches, Texas.

Christian, C.L. 2013. Vegetative community development on reclaimed lignite coal mine land in east Texas. MS thesis. Stephen F. Austin State University, Nacogdoches, Texas.

Diggs, G.M., Jr., B.L. Lipscomb, M.D. Reed, and R.J. O’Kennon. 2006. Illustrated Flora of East Texas, Vol. 1: Introduction, Pteridophytes, Gymnosperms, and Monocotyledons. Botanical Research Institute of Texas, Fort Worth.

Dolezel, R. 1975. Soil Survey of Panola County, Texas. United States Department of Agriculture, Soil Conservation Service, in cooperation with Texas Agricultural Experiment Station.

Gilland, K.E. and B.C. McCarthy. 2014. Microtopography influences early successional plant communities on experimental coal surface mine land reclamation. Restoration Ecology 22:232-239. http://dx.doi.org/10.1111/rec.12066

Grimes, P. 2010. Personal communication. Beckville Mine, Beckville, Texas. Luminant Mining Company.

Gorsira, B. and K.L. Risenhoover. 1994. An evaluation of woodland reclamation on strip-mined lands in east Texas. Environmental Management 18: 787-793. http://dx.doi.org/10.1007/BF02394641

Gould, F.W. 1962. Texas plants, a checklist and ecological summary. The Agricultural and Mechanical College of Texas, Texas Agricultural Experiment Station.

Holl, K.D. 2002. Long-term vegetation recovery on reclaimed coal surface mines in the eastern USA. Journal of Applied Ecology 39: 960-970. http://dx.doi.org/10.1046/j.1365-2664.2002.00767.x 
Holl, K.D. and J. Cairns, Jr. 1994. Vegetational community development on reclaimed coal surface mines in Virginia. Bulletin of the Torrey Botanical Club 121: 327-337. http://dx.doi.org/10.2307/2997006

Kee, D.D. 1984. The effect of cover crop and fertilizer rate on the growth and survival of loblolly pine in east Texas mine spoil. MS thesis. Texas A\&M University, College Station, Texas.

Mask, C.L. 1983. Row seeding of forest tree species on lignite spoils in east Texas. MS thesis. Stephen F. Austin State University, Nacogdoches, Texas.

McCune, B. and M.J. Mefford. 2011. PC-ORD. Multivariate analysis of ecological data. Version 6.0. MjM Software, Gleneden Beach, Oregon.

McGuire, M.A. 1998. Effects of stock type, fall nursery fertilization and ectomycorrhizal inoculation on survival of longleaf pine (Pinus palustris Mill.) seedlings planted on lignite minespoil. MS thesis. Stephen F. Austin State University, Nacogdoches, Texas.

Musgraves, J.R. 1995. Survival of longleaf pine (Pinus palustris Mill.) on mine reclamation sites using different regeneration methods. MS thesis. Stephen F. Austin State University, Nacogdoches, Texas.

NIISS (National Institute of Invasive Species Science). Updated May 25, 2010. Modified Whittaker Plot. Accessed June 4, 2010. http://www.niiss.org/cwis438/websites/niiss/FieldMethods/ModWhit.php?WebSiteID=1

RCT (Railroad Commission of Texas). 2015. Annual coal production (web page link to Excel file). Available online at http://www.rrc.state.tx.us/mining-exploration/programs/surface-coalmining-exploration-program/; last accessed Nov. 6, 2015.

Shupe, M.A. 1986. The effects of nitrogen and phosphorus fertilizer on a young loblolly pine plantation on lignite mine spoil in east Texas. MS thesis. Stephen F. Austin State University, Nacogdoches, Texas.

Skousen, J.G., C.A. Call, and R.W. Knight. 1990. Natural revegetation of an unreclaimed lignite surface mine in east-central Texas. The Southwestern Naturalist 35: 434-440. http://dx.doi.org/10.2307/3672042 
Skousen, J. and C.E. Zipper. 2014. Post-mining policies and practices in the Eastern USA coal region. International Journal of Coal Science \& Technology 1: 135-151. http://dx.doi.org/10.1007/s40789-014-0021-6

Stohlgren, T.J., M.B. Falkner, and L.D. Schell. 1995. A modified-Whittaker nested vegetation sampling method. Vegetatio 117:113-121. http://dx.doi.org/10.1007/BF00045503

Toups, B.G. 1986. Comparison of site quality for loblolly pine on selected mined and non-mined soils in Panola County, Texas. MS thesis. Stephen F. Austin State University, Nacogdoches, Texas.

USDA (United States Department of Agriculture), Natural Resources Conservation Service. 2013. The PLANTS Database (http://plants.usda.gov, 4 March 2013). National Plant Data Team, Greensboro, North Carolina, United States.

Westerman, C.A. 1997. Vegetation trends in reclaimed areas at Gibbons Creek Lignite Mine, Grimes County, Texas. Proceedings American Society of Mining and Reclamation 1997. pp 77-81. http://dx.doi.org/10.21000/JASMR97010077

Wood, G.A. 1985. Two-year survival and growth of loblolly pine seedlings from two Texas seed sources on lignite minesoils. MS thesis. Stephen F. Austin State University, Nacogdoches, Texas.

Zipper, C.E., J.A. Burger, J.G. Skousen, P.N. Angel, C.D. Barton, V. Davis, and J.A. Franklin. 2011. Restoring forests and associated ecosystem services on Appalachian coal surface mines. Environmental Management 47: 751-765. http://dx.doi.org/10.1007/s00267-011-9670-z 


\section{Appendix 1.}

Table A-1. Species planted on mixed pine-hardwood reclamation sites from 1980 to 2009 on Beckville lignite coal surface mine in Panola County, Texas.

\begin{tabular}{|c|c|c|c|c|c|c|c|c|c|c|c|c|c|c|c|c|c|c|c|c|c|c|c|c|c|c|c|c|c|c|}
\hline Scientific name & 80 & 81 & 82 & 83 & 84 & 85 & 86 & 87 & 88 & 89 & 90 & 91 & 92 & 93 & 94 & 95 & 96 & 97 & 98 & 99 & $\mathbf{0 0}$ & 01 & $\mathbf{0 2}$ & $\mathbf{0 3}$ & 04 & 05 & 06 & $\mathbf{0 7}$ & $\mathbf{0 8}$ & 09 \\
\hline Acer rubrum & & & & & & $\bullet$ & & & & & & & & & & & & & & & & $\bullet$ & $\bullet$ & $\bullet$ & & & & & & \\
\hline Betula nigra & & & $\bullet$ & $\bullet$ & $\bullet$ & • & & • & $\bullet$ & & • & & & & $\bullet$ & & & & & & & $\bullet$ & & $\bullet$ & $\bullet$ & $\bullet$ & • & • & & \\
\hline $\begin{array}{l}\text { Callicarpa } \\
\text { americana }\end{array}$ & & & & & & & & & & & & & & & & $\bullet$ & & & & & & - & & & & • & • & & - & \\
\hline Carya aquatica & & & & & & & & & & & & & & & & $\bullet$ & & & & & & $\bullet$ & $\bullet$ & $\bullet$ & $\bullet$ & - & $\bullet$ & $\bullet$ & • & $\bullet$ \\
\hline $\begin{array}{l}\text { Carya } \\
\text { illinoinensis }\end{array}$ & & & & & & & & & & & $\bullet$ & $\bullet$ & & $\bullet$ & $\bullet$ & $\bullet$ & $\bullet$ & - & $\bullet$ & $\bullet$ & $\bullet$ & $\bullet$ & $\bullet$ & $\bullet$ & $\bullet$ & $\bullet$ & • & - & • & $\bullet$ \\
\hline Catalpa sp. & • & & • & & & & • & - & $\bullet$ & $\bullet$ & & $\bullet$ & • & • & • & & & & & & & & & & & & & & & \\
\hline $\begin{array}{l}\text { Celtis laevigata } \\
\text { Cercis }\end{array}$ & & & & & & & & & • & & & $\bullet$ & & & & & & & & & & $\bullet$ & & & & $\bullet$ & - & - & & \\
\hline canadensis & & & & & & & & & & & & & & & & & & & & & & $\bullet$ & $\bullet$ & & $\bullet$ & $\bullet$ & $\bullet$ & - & $\bullet$ & \\
\hline $\begin{array}{l}\text { Diospyros } \\
\text { virginiana }\end{array}$ & & & & & & $\bullet$ & - & - & $\bullet$ & & & $\bullet$ & • & - & $\bullet$ & $\bullet$ & $\bullet$ & - & $\bullet$ & & $\bullet$ & $\bullet$ & - & $\bullet$ & $\bullet$ & - & • & • & • & $\bullet$ \\
\hline Elaeagnus sp. & & • & - & - & & & • & • & $\bullet$ & & & & & & & & & & & & & & & & & & & & & \\
\hline $\begin{array}{l}\text { Fraxinus } \\
\text { pennsylvanica }\end{array}$ & $\bullet$ & & $\bullet$ & $\bullet$ & • & $\bullet$ & • & • & • & $\bullet$ & & $\bullet$ & • & - & $\bullet$ & $\bullet$ & $\bullet$ & - & $\bullet$ & • & $\bullet$ & & & & & & $\bullet$ & $\bullet$ & • & $\bullet$ \\
\hline Ilex vomitoria & & & & & & & & & & & & & & & $\bullet$ & $\bullet$ & & & & & & $\bullet$ & & $\bullet$ & & & & & & \\
\hline Juglans nigra & & & & & & - & & & & & & & & & & & & & & & & $\bullet$ & • & - & • & • & • & • & • & \\
\hline $\begin{array}{l}\text { Juniperus } \\
\text { virginiana }\end{array}$ & & & & & & & & & & & & - & & & & & • & & & & & • & & • & & & & & & \\
\hline Lespedeza sp. & & & & & & - & - & & $\bullet$ & & & & & & • & • & $\bullet$ & - & & • & • & & & & & & & & & \\
\hline $\begin{array}{l}\text { Liquidambar } \\
\text { styraciflua }\end{array}$ & & & & & - & - & - & - & - & $\bullet$ & & - & - & - & & $\bullet$ & & & & & & & & & & & & & & \\
\hline Morella cerifera & & & & & & & & & & & & & & & & $\bullet$ & & • & $\bullet$ & & • & & $\bullet$ & $\bullet$ & $\bullet$ & • & & $\bullet$ & • & • \\
\hline $\begin{array}{l}\text { Morus spp. } \\
\text { Pinus echinata }\end{array}$ & $\bullet$ & $\bullet$ & & & & & & & & $\bullet$ & & - & • & • & & & & & & & & & & & & & & & & \\
\hline $\begin{array}{l}\text { Pinus taeda } \\
\text { Platanus } \\
\text { occidentalis }\end{array}$ & $\bullet$ & • & - & • & • & - & - & - & $\bullet$ & $\bullet$ & - & • & & & $\bullet$ & & $\bullet$ & - & • & • & $\bullet$ & $\bullet$ & $\bullet$ & $\bullet$ & - & $\bullet$ & $\bullet$ & • & $\bullet$ & $\bullet$ \\
\hline $\begin{array}{l}\text { Populus deltoides } \\
\text { Prunus } \\
\text { angustifolia }\end{array}$ & • & • & $\bullet$ & & • & • & • & • & • & • & • & • & • & • & • & • & • & • & • & & • & & • & • & • & • & & & • & - \\
\hline
\end{tabular}


Table A-1, continued.

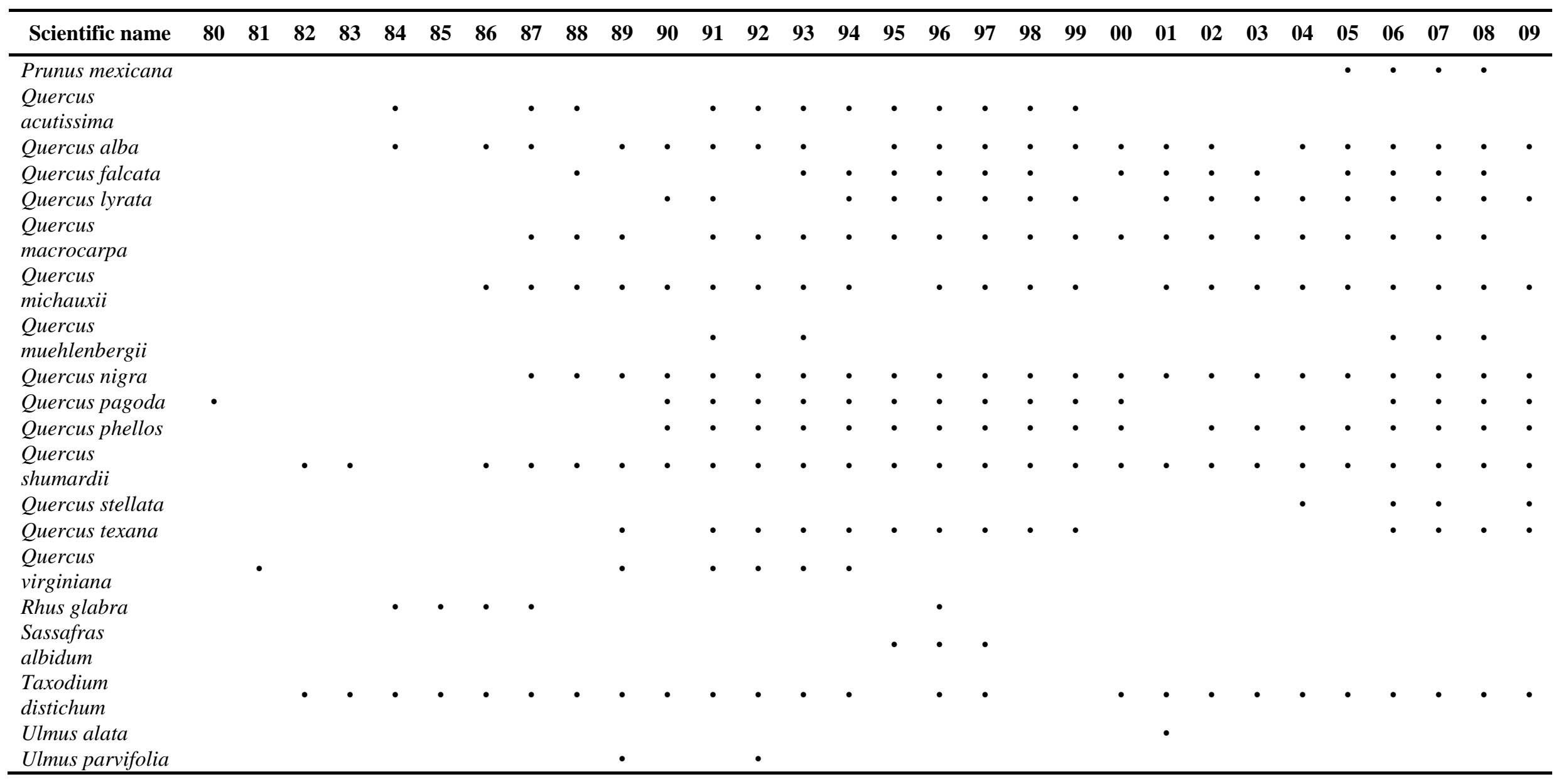


Table A-2. Species codes utilized in ordination graphs (Figs. 2, 3, 4, 7, 8, 9).

\begin{tabular}{|c|c|c|}
\hline Species code & Scientific name & Common name \\
\hline ACENEG & Acer negundo $\mathrm{L}$. & Boxelder \\
\hline ACERUB & Acer rubrum $\mathrm{L}$. & Red maple \\
\hline AMBART & Ambrosia artemisiifolia $\mathrm{L}$. & Annual ragweed \\
\hline AMPARB & Ampelopsis arborea (L.) Koehne & Peppervine \\
\hline BACHAL & Baccharis halimifolia $\mathrm{L}$. & Eastern baccharis \\
\hline BERSCA & Berchemia scandens (Hill) K. Koch & Alabama supplejack \\
\hline BROMSP & Bromus sp. L. & Brome \\
\hline CARILL & Carya illinoinensis (Wangenh.) K. Koch & Pecan \\
\hline CATASP & Catalpa sp. Scop. & Catalpa \\
\hline CELLAE & Celtis laevigata Willd. & Sugarberry \\
\hline CENPUL & Centaurium pulchellum (Sw.) Druce & Branched centaury \\
\hline CHASES & Chasmanthium sessiliflorum (Poir.) Yates & Longleaf woodoats \\
\hline CYNDAC & Cynodon dactylon (L.) Pers. & Coastal bermudagrass \\
\hline DAUCAR & Daucus carota $\mathrm{L}$. & Queen Anne's lace \\
\hline DESILL & $\begin{array}{l}\text { Desmanthus illinoensis (Michx.) MacMill. ex B.L. } \\
\text { Rob \& Fernald }\end{array}$ & Illinois bundleflower \\
\hline DICHSP & Dichanthelium sp. (Hitchc. \& Chase) Gould & Rosette grass \\
\hline DIOVIR & Diospyros virginiana $\mathrm{L}$. & Common persimmon \\
\hline FRAPEN & Fraxinus pennsylvanica Marshall & Green ash \\
\hline GALAPA & Galium aparine $\mathrm{L}$. & Catchweed bedstraw \\
\hline GAMOSP & Gamochaeta sp. Weddell & Everlasting \\
\hline GERCAR & Geranium carolinianum $\mathrm{L}$. & Carolina geranium \\
\hline GLETRI & Gleditsia triacanthos $\mathrm{L}$. & Honeylocust \\
\hline HETSUB & Heterotheca subaxillaris (Lam.) Britton \& Rusby & Camphorweed \\
\hline ILEVOM & Ilex vomitoria Aiton & Yaupon \\
\hline JUGNIG & Juglans nigra $\mathrm{L}$. & Black walnut \\
\hline JUNVIR & Juniperus virginiana $\mathrm{L}$. & Eastern redcedar \\
\hline LESPSP & Lespedeza sp. Michx. & Lespedeza \\
\hline LIGUSP & Ligustrum sp. L. & Privet \\
\hline LIQSTY & Liquidambar styraciflua $\mathrm{L}$. & Sweetgum \\
\hline LOLISP & Lolium sp. L. & Ryegrass \\
\hline LONJAP & Lonicera japonica Thunb. & Japanese honeysuckle \\
\hline MELALB & Melilotus alba & Sweetclover \\
\hline PANVIR & Panicum virgatum $\mathrm{L}$. & Switchgrass \\
\hline PARQUI & Parthenocissus quinquefolia (L.) Planch. & Virginia creeper \\
\hline PINECH & Pinus echinata Mill. & Shortleaf pine \\
\hline PINTAE & Pinus taeda $\mathrm{L}$. & Loblolly pine \\
\hline PLAOCC & Platanus occidentalis L. & American sycamore \\
\hline POPDEL & Populus deltoides W. Bartram ex Marshall & Eastern cottonwood \\
\hline PRUANG & Prunus angustifolia Marshall & Chickasaw plum \\
\hline QUEALB & Quercus alba $\mathrm{L}$. & White oak \\
\hline QUEFAL & Quercus falcata Michx. & Southern red oak \\
\hline QUELYR & Quercus lyrata Walter & Overcup oak \\
\hline QUEMAC & Quercus macrocarpa Michx. & Bur oak \\
\hline QUEMIC & Quercus michauxii Nutt. & Swamp chestnut oak \\
\hline QUENIG & Quercus nigra $\mathrm{L}$. & Water oak \\
\hline QUEPAG & Quercus pagoda Raf. & Cherrybark oak \\
\hline QUEPHE & Quercus phellos L. & Willow oak \\
\hline QUERSP & Quercus sp. L. & Oak \\
\hline
\end{tabular}


Table A-2, continued.

\begin{tabular}{lll}
\hline \multicolumn{1}{c}{ Species code } & \multicolumn{1}{c}{ Scientific name } & \multicolumn{1}{c}{ Common name } \\
\hline QUESHU & Quercus shumardii Buckley & Shumard's oak \\
RUBUSP & Rubus sp. L. & Blackberry \\
RUDHIR & Rudbeckia hirta L & Blackeyed Susan \\
RUMCRI & Rumex crispus L. & Curly dock \\
SCHSCO & Schizachyrium scoparium (Michx.) Nash & Little bluestem \\
SMIBON & Smilax bona-nox L. & Saw greenbrier \\
SMIROT & Smilax rotundifolia L. & Common greenbrier \\
SOLASP & Solanum sp. & Nightshade \\
SOLCAN & Solidago canadensis L. & Canada goldenrod \\
SORHAL & Sorghum halepense (L.) Pers. & Johnsongrass \\
STEMED & Stellaria media (L.) Vill. & Common chickweed \\
TRIFSP & Trifolium sp. L. & Clover \\
TRIVES & Trifolium vesiculosum Savi & Arrowleaf clover \\
TOXRAD & Toxicodendron radicans (L.) Kuntze & Poison ivy \\
ULMALA & Ulmus alata Michx. & Winged elm \\
ULMPAR & Ulmus parvifolia Jacq. & Chinese elm \\
VICISP & Vicia sp. L. & Vetch \\
VICVIL & Vicia villos Roth & Winter vetch \\
VITROT & Vitis rotundifolia Michx. & Muscadine \\
\hline
\end{tabular}


Appendix 2. Selected photos of reclaimed sites planted in mixed-pine hardwood and unmined forested reference sites on Beckville lignite coal surface mine in Panola County, Texas.

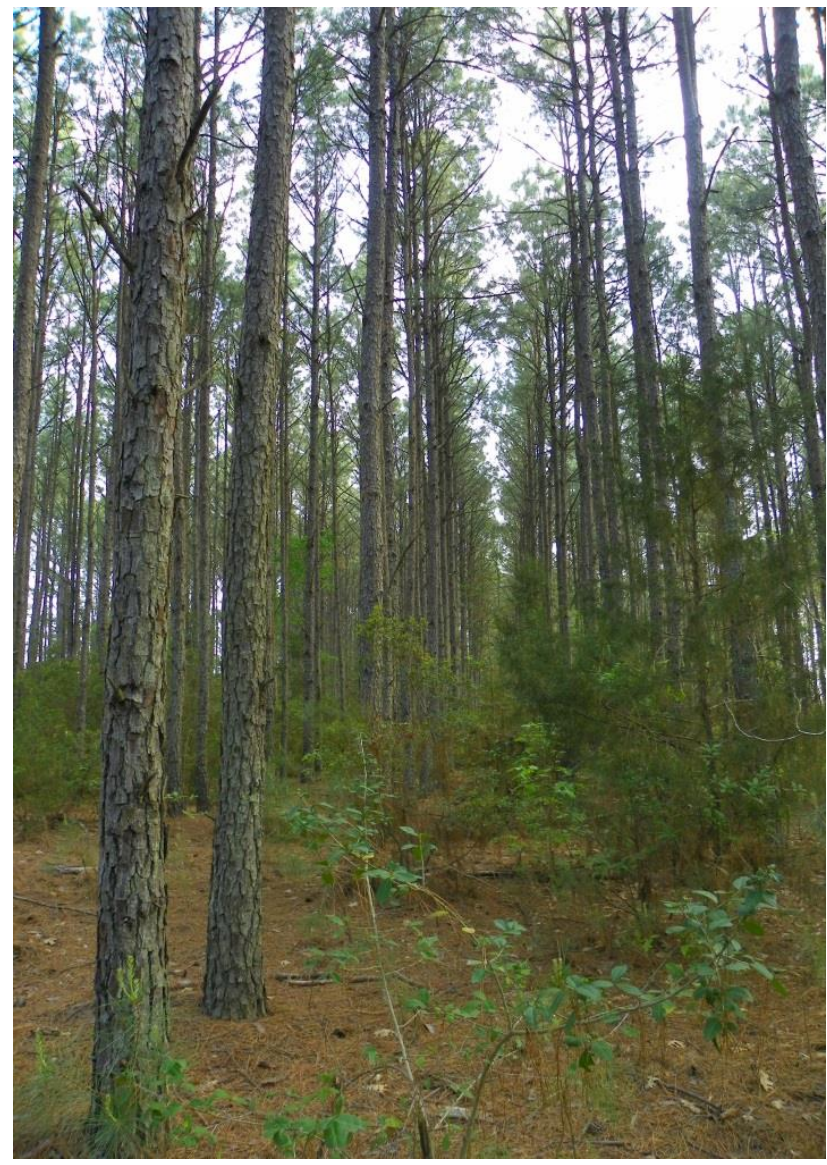

Figure A2.1. Mixed site planted in 1982 (28 years old).

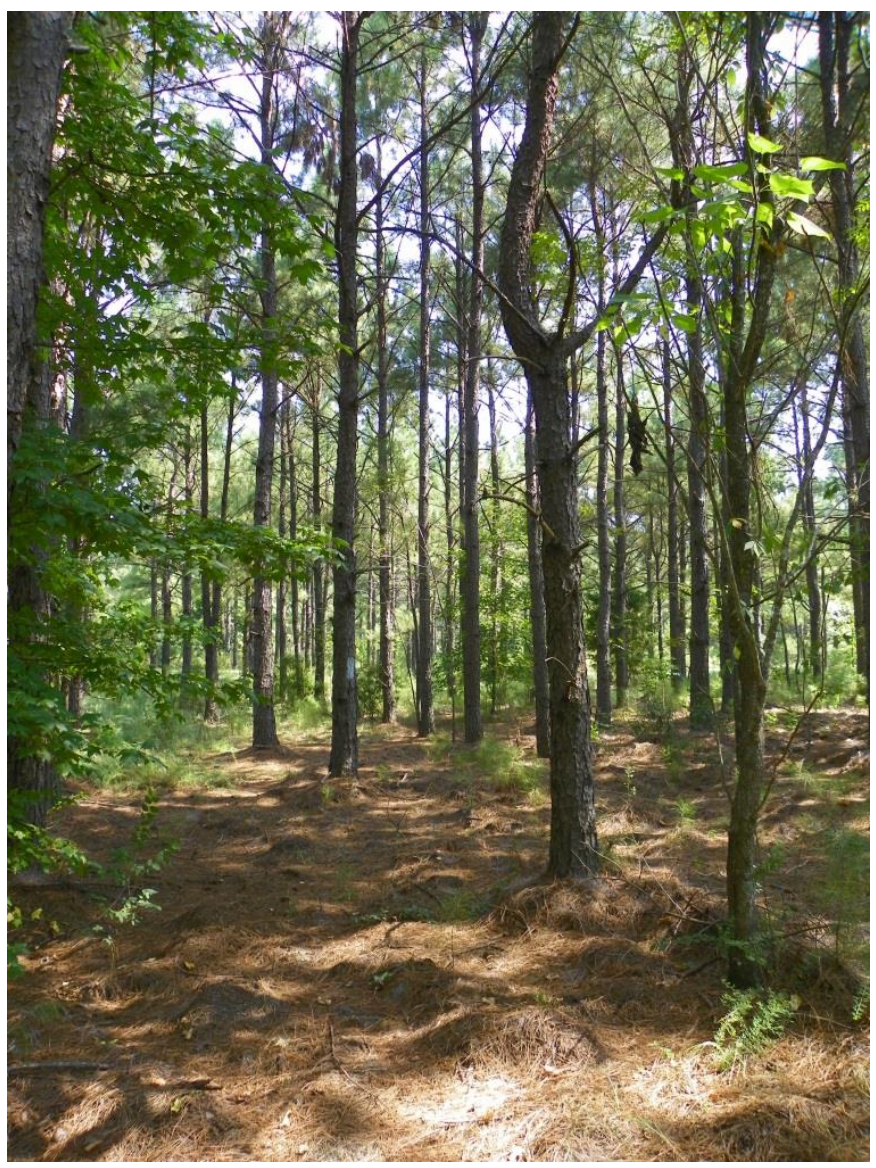

Figure A2.2. Mixed site planted in 1986 (24 years old). 
Appendix 2, continued.

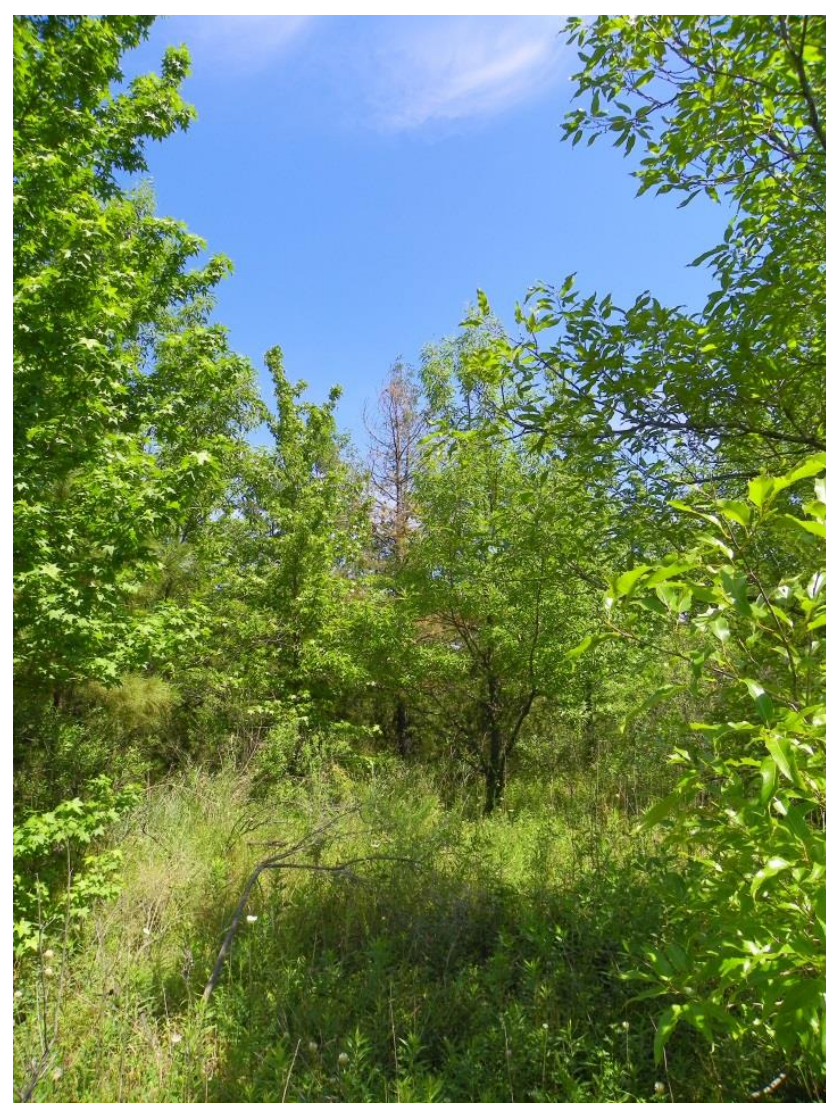

Figure A2.3. Mixed site planted in 1987 (23 years old).

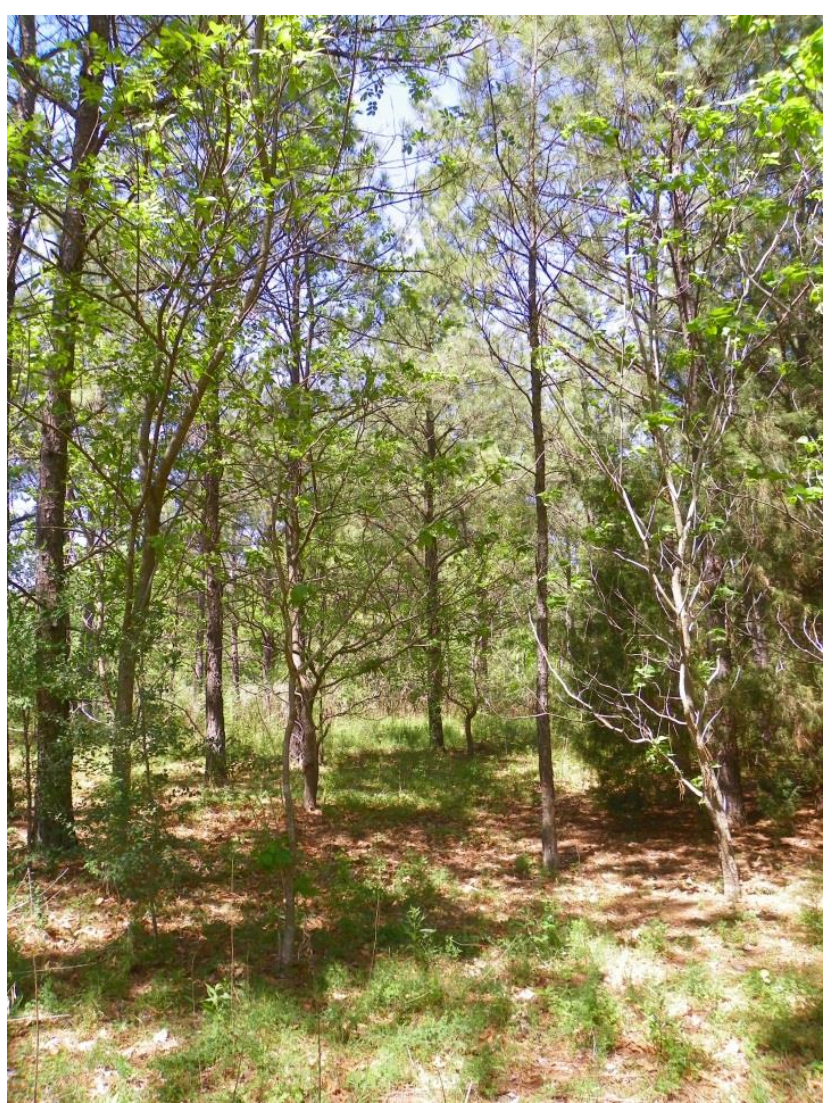

Figure A2.4. Mixed site planted in 1987 (23 years old). 
Appendix 2, continued.

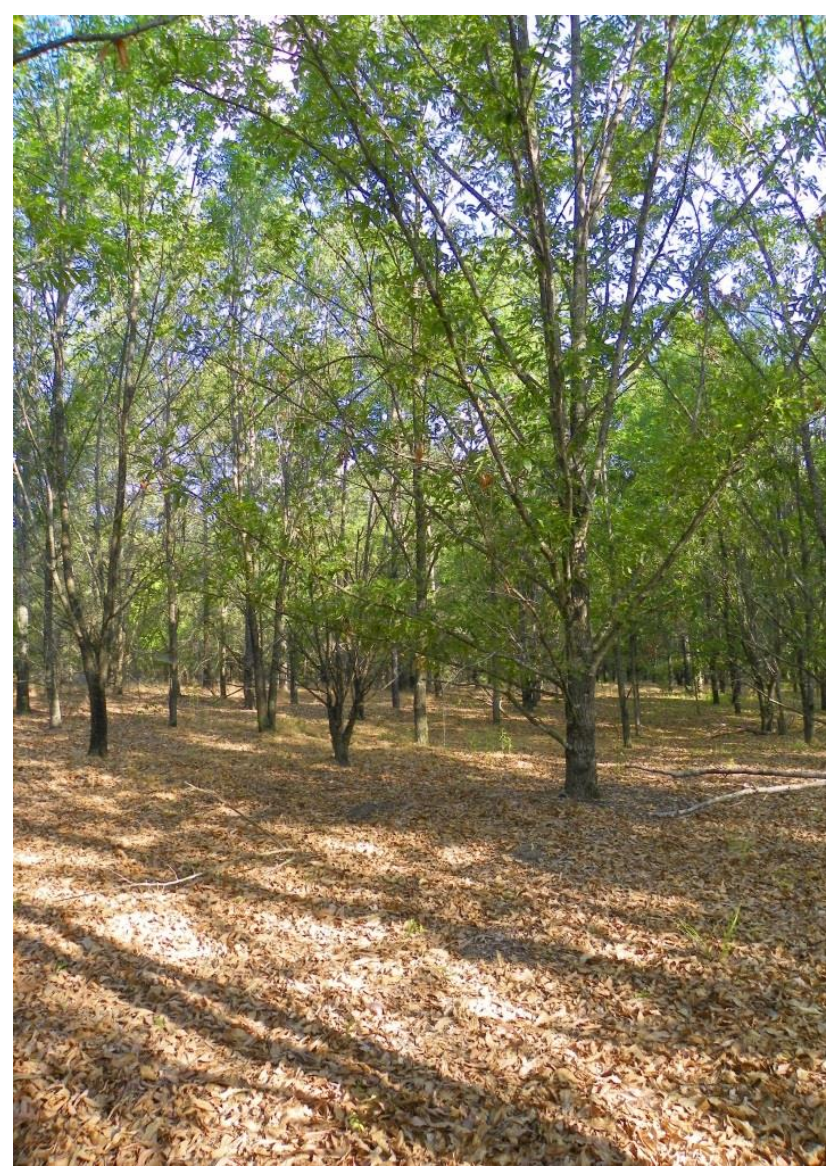

Figure A2.5. Mixed site planted in 1992 (18 years old).

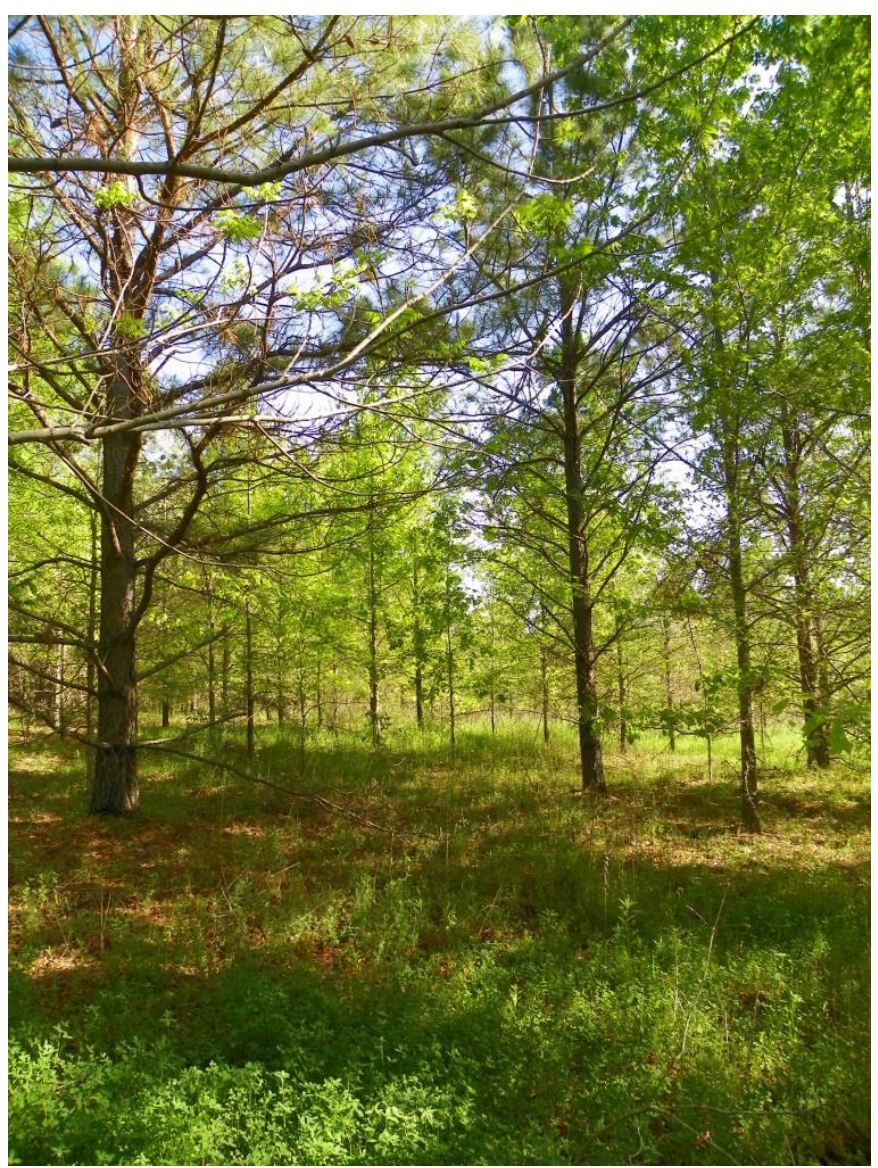

Figure A2.6. Mixed site planted in 1995 (15 years old). 
Appendix 2, continued.

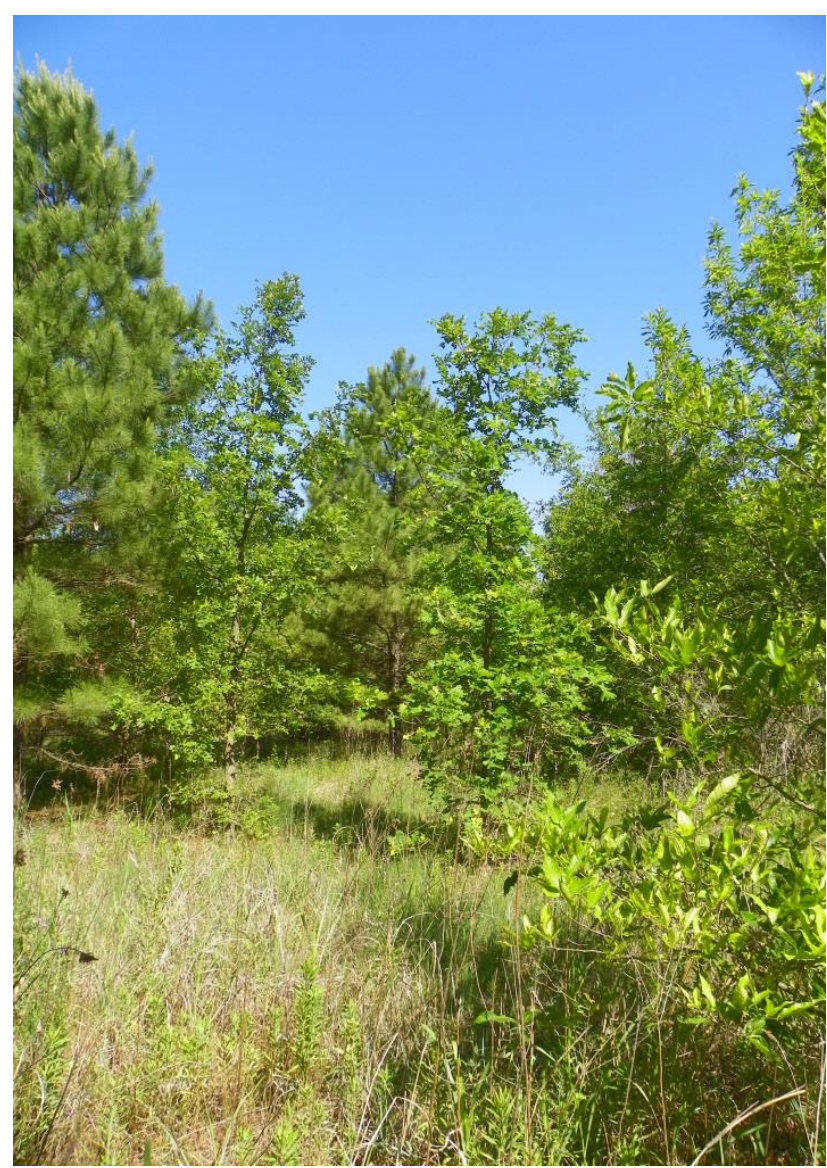

Figure A2.7. Mixed site planted in 1997 (13 years old).

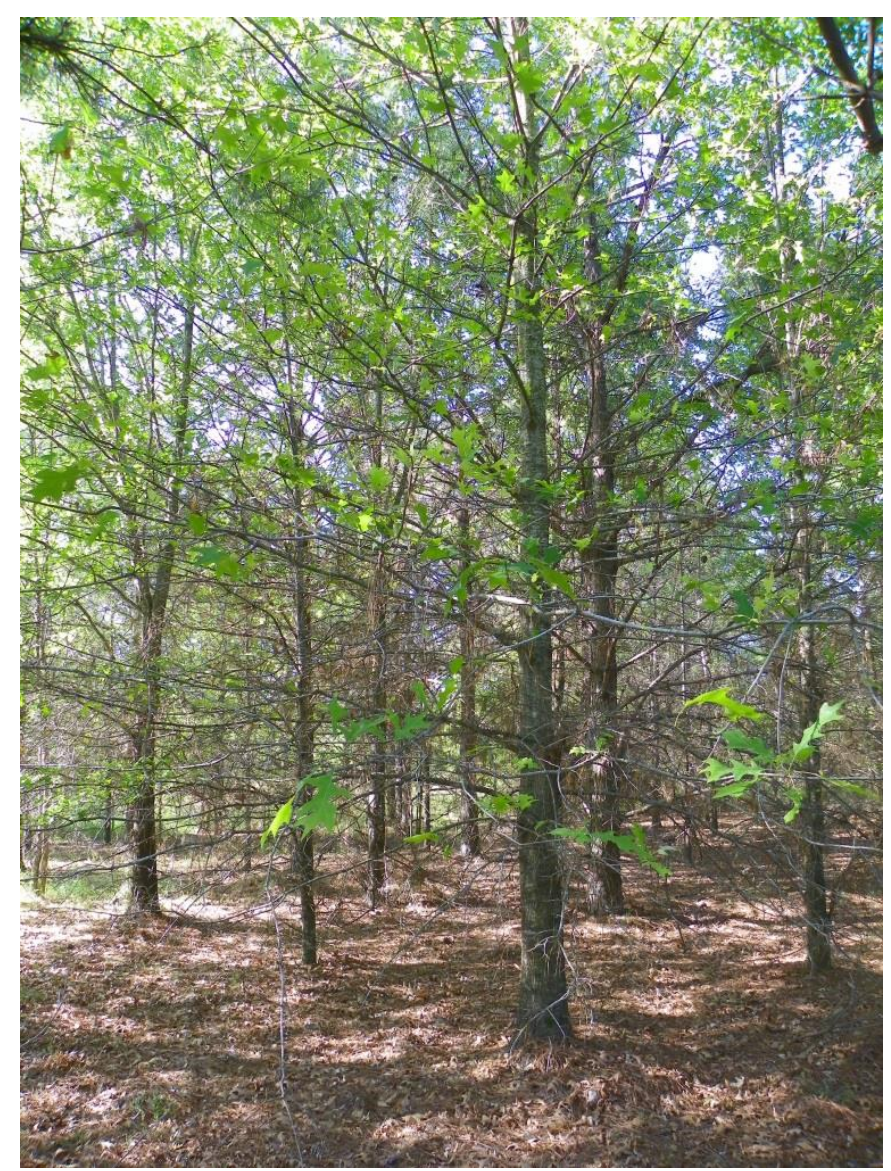

Figure A2.8. Mixed site planted in 1998 (12 years old). 
Appendix 2, continued.

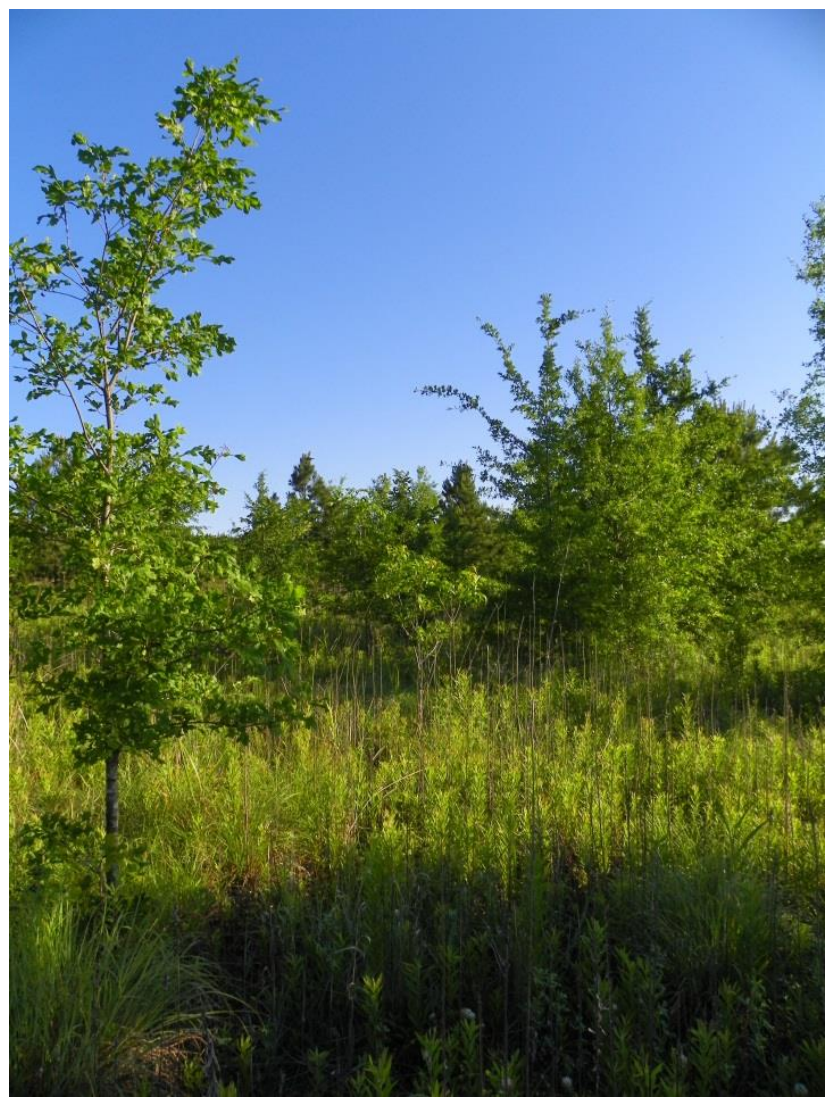

Figure A2.9. Mixed site planted in 2001 (9 years old).

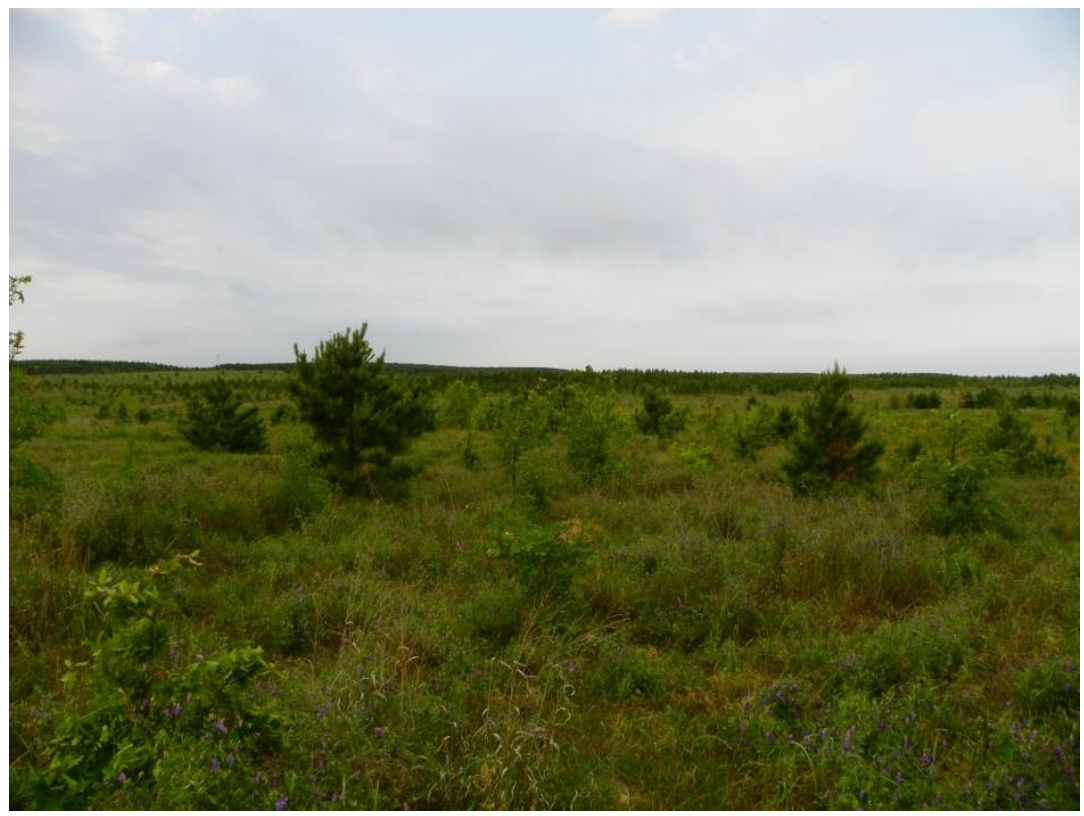

Figure A2.10. Mixed site planted in 2005 (5 years old). 
Appendix 2, continued.

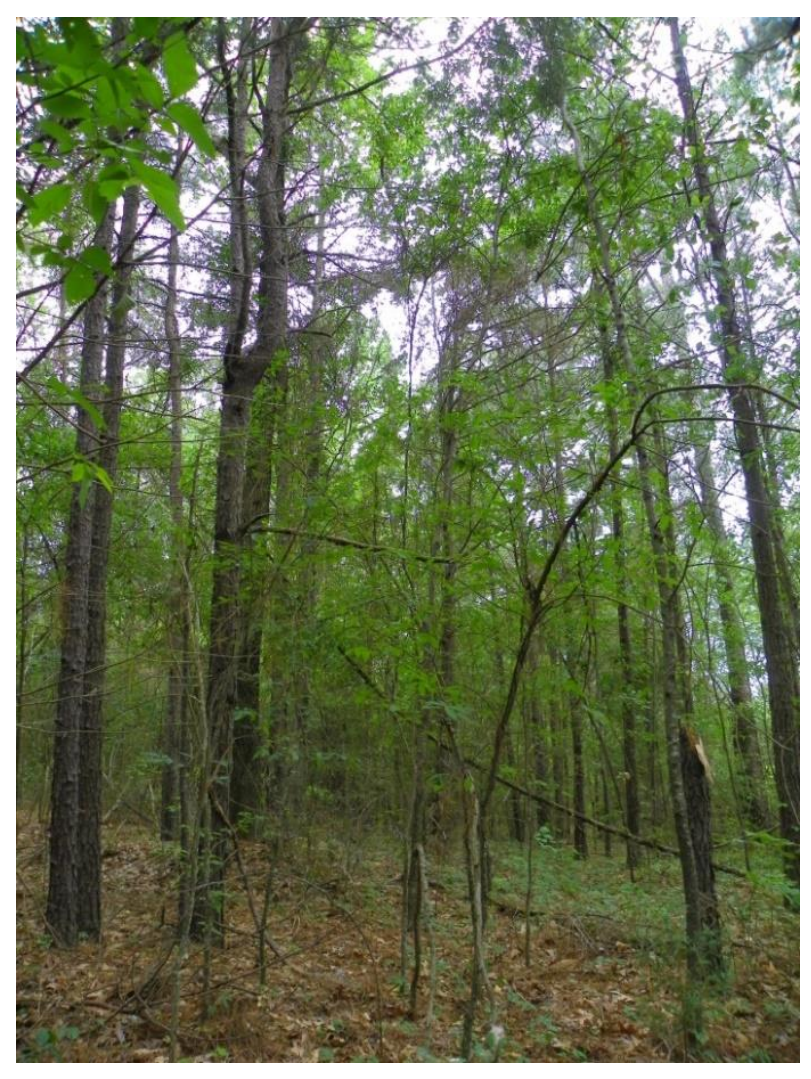

Figure A2.11. Reference site.

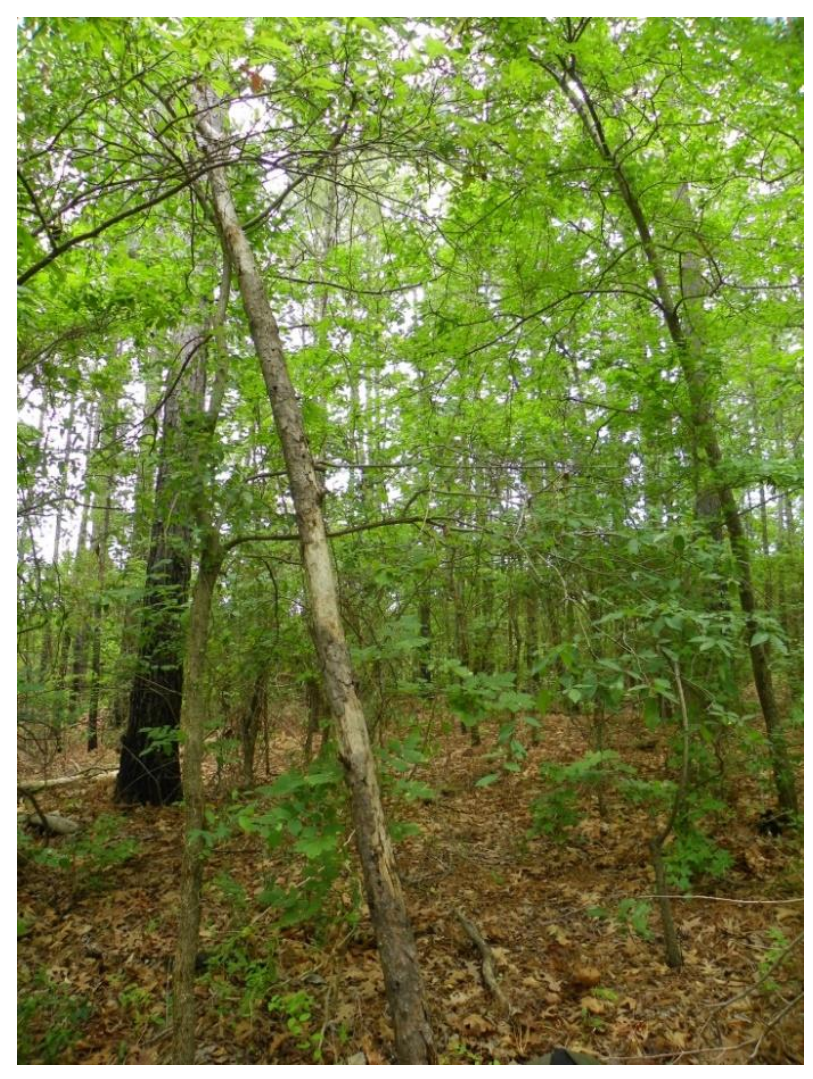

Figure A2.12. Reference site. 